Research Article

\title{
Tough and Fatigue-Resistant Hydrogels with Triple Interpenetrating Networks
}

\author{
Jilong Wang $\mathbb{D},{ }^{1,2}$ Junhua Wei $\mathbb{D},{ }^{2}$ Siheng Su, ${ }^{3}$ and Jingjing Qiu $\mathbb{C}^{2}$ \\ ${ }^{1}$ Key Laboratory of Textile Science \& Technology of Ministry of Education, College of Textiles, Donghua University, \\ Shanghai 201620, China \\ ${ }^{2}$ Department of Mechanical Engineering, Texas Tech University, 2500 Broadway, P.O. Box 43061, Lubbock, TX 79409, USA \\ ${ }^{3}$ Department of Mechanical Engineering, California State University Fullerton, 800N State College Blvd, Fullerton, CA 92831, USA
}

Correspondence should be addressed to Jingjing Qiu; jenny.qiu@ttu.edu

Received 24 June 2018; Revised 10 October 2018; Accepted 27 November 2018; Published 25 February 2019

Academic Editor: Xuping Sun

Copyright (c) 2019 Jilong Wang et al. This is an open access article distributed under the Creative Commons Attribution License, which permits unrestricted use, distribution, and reproduction in any medium, provided the original work is properly cited.

\begin{abstract}
Biomimetic hydrogels with triple networks have been developed via in situ polymerization and addition of graphene oxide (GO) nanosheets, which achieve improved toughness and superior fatigue resistance, simultaneously. Compared with pristine calcium alginate/polyacrylamide double network (DN) hydrogels, the integration of a calcium-induced graphene oxide network enhances the crosslinking degree of triple network (TN) hydrogels with improved compressive strength by $172 \%$ and toughness by $174 \%$. In addition, cyclic compressive loading-unloading curves depict excellent fatigue resistance because of reversible calcium alginate and calcium-induced GO networks, whereas high strength and toughness of traditional DN gels derive from the first sacrificial network, which leads to inferior fatigue resistance. Toughness of these TN gels was still kept at $110 \mathrm{~kJ} \mathrm{~m}^{-3}$ at the fifth cycle which is equal to that of articular cartilages. The swelling property of these DN and TN hdyrogels is also systematically explored, which exhibits that GO can reduce the swelling to maintain the mechanical properties of TN gels. The internal fracture mechanisms of these TN hydrogels are studied via swelling tests of precompressed and as-prepared gels. These synergistic effects of the reversible ions crosslinking polymer network and nanofillers open a new platform to design supertough and fatigue-resistant hydrogels. In addition, these TN hydrogels are talented replacements for load-bearing parts, like cartilage due to its high toughness and superior fatigue resistance.
\end{abstract}

\section{Introduction}

The native cartilage is an important part in the human body to carry regular articular functions by adsorbing impact energy, distributing loads, and reducing friction [1-4]. Traditional approaches including microfracture therapy and periosteal grafting may lead to repeated injury due to poor functions [5-7]. Therefore, an emerging therapy to repair or replace the injured cartilage by using artificial cartilage has attracted large amounts of attention due to its advantages like easy implantation and high biocompatibility [5].

Gels are one of promising candidates as scaffolds in tissue engineering. Double network (DN) gels combining two independently crosslinked networks are the common method to achieve tough hydrogels $[8,9]$. However, limited fatigue resistance extremely restricts its clinical applications $[10,11]$.
Alginate hydrogels, ionically crosslinked via divalent or trivalent cations, have been extensively applied in bone tissue engineering and wound dressing [12, 13], which exhibits excellent reversibility [14]. Darnell and his coworkers first developed tough and extensible calcium alginate/polyacrylamide (PAm) double network hydrogels by blending calcium alginate networks and PAm networks [15]. In addition, the biocompatibility of calcium alginate/PAm gels was also investigated, which suggests these gels are biocompatible [16]. Li et al. successfully developed improved calcium alginate/PAm gels via soaking hydrogels in $\mathrm{CaCl}_{2}$ solution [17]. Yang et al. systematically investigated the mechanical properties of diverse multivalent cation crosslinked alginate/PAm gels [18]. Recently, Hong et al. proposed an assumption that the reversible calcium ionically crosslinked alginate network adsorbs dissipation energy, whereas the covalently 
crosslinked PAm network preserves elasticity during loading [19], which demonstrates that ionically crosslinked alginate-based DN gels achieve superior fatigue resistance. However, compared to other DN hydrogels, the calcium alginate network improved DN hydrogels which present inferior mechanical properties. So, to enhance the mechanical property of these DN gels with high fatigue resistance is meaningful.

On the other hand, nanocomposite hydrogels are considered as a simple approach to enhance the mechanical performance via introduction of various reinforced fillers such as cellulose, silica nanoparticles, carbon nanotubes, and graphene oxide (GO) [20-26]. Due to the giant specific surface area and enormous functional groups including hydroxyl, epoxide, and carboxyl groups, GO has received huge attention to improve mechanical properties of hydrogels $[27,28]$. Previous studies have been performed to develop graphene nanosheet-reinforced polyacrylamide networks to increase mechanical behaviors of PAm hydrogels [29-31]. Cong et al. developed calcium-GO/PAm hydrogels with excellent elasticity [32]. In addition, He et al. fabricated GO/sodium alginate (SA) hydrogel fibers via the wet-spinning technique. After cross-linking by calcium ions, the mechanical performance of GO/sodium alginate hydrogel-based fibers were significantly improved [33]. Fan et al. designed GO/SA/PAm hydrogels to absorb dyes with large adsorption ability [34].

To achieve tough hydrogels with superior fatigue resistance, triple network (TN) hydrogels were built by combining calcium-induced graphene oxide (GO) physical networks, calcium ionically crosslinked alginate networks, and covalently crosslinked polyacrylamide networks. A mixed mechanism was also developed to explain tough and fatigue-resistant TN hydrogels. The calcium ionically crosslinked alginate network and calcium-induced GO networks consume mechanical energy, while covalently crosslinked PAm networks sustain elasticity during deformation. In addition, nanofillers with a large specific area and functional groups interpenetrate with PAm chains and bonds to alginate and PAm chains, which improve the mechanical property of gels. With the addition of GO nanosheets, the mechanical performance of TN hydrogels has been largely improved. In addition, cyclic compressive loading-unloading curves show magnificent fatigue resistance of TN hydrogels. Furthermore, the swelling behavior of DN and TN gels has been well displayed, which exhibits that GO can restrain the swelling of the PAm network leading to superior mechanical performance in swelling state. Also, swelling tests have been performed to analyze the internal fracture mechanism of these hydrogels. These TN hydrogels develop a new method to design tough and fatigue-resistant hydrogels, which may be a promising substitute for load-bearing tissues.

\section{Experimental}

2.1. Materials. Graphite was provided from Asbury Carbons. Sodium alginate $(\mathrm{A})$ was nicely provided from FMC BioPolymer. Other chemicals including acrylamide (Am), $\mathrm{CaCl}_{2}$, $\mathrm{HNO}_{3}$, hydrogen chloride, $\mathrm{NaClO}_{3}$, ammonium persulfate
TABLE 1: The main component of various hydrogels.

\begin{tabular}{lccc}
\hline Sample label & $\begin{array}{c}\text { Graphene oxide } \\
(\mathrm{mg})\end{array}$ & $\begin{array}{c}\text { Sodium alginate } \\
(\mathrm{mg})\end{array}$ & $\begin{array}{c}\text { Acrylamide } \\
(\mathrm{mg})\end{array}$ \\
\hline SN & 0 & 0 & 1200 \\
DN1 & 0 & 200 & 1200 \\
DN2 & 0 & 400 & 1200 \\
TN1 & 50 & 200 & 1200 \\
TN2 & 100 & 200 & 1200 \\
TN3 & 100 & 400 & 1200 \\
TN4 & 100 & 200 & 1200 \\
\hline
\end{tabular}

(APS), and $\mathrm{N}, \mathrm{N}^{\prime}$-methylenebis(acrylamide) (MBAA) were all provided by Sigma-Aldrich.

2.2. Fabrication of Graphene Oxide (GO). GO was achieved via a modified Brodie method, which has been introduced in our previous work [35]. Specifically, $2 \mathrm{~g}$ of graphite was blended with $17 \mathrm{~g}$ of $\mathrm{NaClO}_{3}$, then $80 \mathrm{~mL}$ of $\mathrm{HNO}_{3}$ was added slowly to avoid burning. After stirring at $60^{\circ} \mathrm{C}$ for 6 hours and then stirring for another 12 hours at room temperature, the diluted $\mathrm{HCl}$ solution and deionized water were used to wash the mixture solution to remove impurities, separately. The resultant was dried to obtain a GO powder by a heater at $70^{\circ} \mathrm{C}$. $1 \mathrm{wt} \%$ and $0.5 \mathrm{wt} \%$ of GO solution were prepared via addition of $100 \mathrm{mg}$ and $50 \mathrm{mg}$ GO powder into $10 \mathrm{~mL}$ deionized water via tip sonication for 3 hours with $100 \mu \mathrm{L}$ ammonia solution.

2.3. Preparation of DN and TN Gels. TN gels were obtained by a previous method [35]. $200 \mathrm{mg}$ of A and $1200 \mathrm{mg}$ of Am were introduced into the GO solution via stirring to achieve a homogenous mixture. After that, $3 \mathrm{mg}$ of MBAA and $36 \mathrm{mg}$ of APS were added into the mixture in an ice bath. The final mixture was transferred into cylinder molds. After heating in an oven for 3 hours, hydrogels were moved into $8 \mathrm{wt} \% \mathrm{CaCl}_{2}$ solution for 2 hours to obtain TN hydrogels. In this manuscript, seven different samples were developed via this process. The content of various samples was collected as shown in Table 1.

\subsection{Characterization}

2.4.1. Material Characterization. An X-ray photoelectron spectroscope (XPS, PHI 5000 VersaProbe), Hitachi 8100 transmission electron microscope (TEM), Siemen AXS D5005 X-ray diffractometer (XRD), thermogravimetric analyzer (TGA), energy-dispersive X-ray analyzer (EDXA), Raman spectrum via a SENTERRA microscope, and Nicolet iS10 Fourier transform infrared (FT-IR) spectrometer were employed to characterize GO.

2.4.2. Compressive Measurements. Instron 5966 was used to measure cylindrical samples, and the velocity was $600 \mu \mathrm{m} / \mathrm{min}$; strain $\varepsilon$ is defined as

$$
\varepsilon=\frac{h}{h_{0}},
$$


where $h$ is the height during deformation and $h_{0}$ is the initial height of the gel sample. The normal stress $\sigma_{\text {normal }}$ is defined as

$$
\sigma_{\text {normal }}=\frac{F}{A_{0}},
$$

where $F$ and $A_{0}$ are defined as the applied load and original top surface area of samples, respectively. The true compressive stress that presents forces per cross-sectional area is achieved as [36]

$$
\sigma_{\text {true }}=\sigma_{\text {normal }}
$$

where $\lambda$ is the deformation ratio which is defined as

$$
\lambda=\frac{h}{h_{0}} .
$$

Five successive loading-unloading cycles were applied to characterize fatigue resistance of achieved gels. Three various strains $(30 \%, 60 \%$, and $90 \%)$ were chosen in this experiment; compressive toughness $\left(U_{\mathrm{CT}}\right)$ is described as

$$
U_{\mathrm{CT}}=\frac{\int_{0}^{0.9} F d s}{\pi r^{2}},
$$

where $s$ is defined as the corresponding displacement during the test, and hysteresis energy $\left(U_{\text {hyst }}\right)$ is calculated as

$$
U_{\text {hyst }}=\frac{\int_{0}^{S_{\text {loading }}} F d s-\int_{0}^{S_{\text {unloading }}} F d s}{\pi r^{2}} .
$$

2.4.3. Swollen Tests of Hydrogels. The swollen ratio (SR) was defined as

$$
\mathrm{SR}=\frac{W_{s}-W_{o}}{W_{o}} * 100 \%,
$$

where $W_{s}$ and $W_{o}$ represent the weight of hydrogels after being swollen in deionized water with time changing and weight of hydrogels before swelling, respectively.

The equilibrium swelling degree (ESD) is defined as [37]

$$
\mathrm{ESD}=\frac{W_{e}-W_{d}}{W_{d}},
$$

where $W_{e}$ is the weight of dried samples and $W_{d}$ means the weight of samples in swollen equilibrium state.

\section{Results and Discussions}

3.1. Material Characterization of Graphene Oxide (GO). GO was achieved via the same method in our previous literature [35] and well dispersed in an aqueous solution because of the oxygen-related functional groups on its surfaces. As shown in Figure 1(a), the obvious peaks around 1300, 1600, 1710, and $3400 \mathrm{~cm}^{-1}$ indicate $\mathrm{C}-\mathrm{O}$, aromatics of graphene $(\mathrm{C}=\mathrm{C}), \mathrm{C}=\mathrm{O}$, and $\mathrm{O}-\mathrm{H}$ groups, respectively, demonstrating that $\mathrm{GO}$ owns a high amount of oxygen [35]. As depicted in Figure 1(b), a high-resolution spectrum of $\mathrm{C} 1$ s exhibits a noticeable degree of oxidation with four main peaks. The peaks around $283.8 \mathrm{eV}, 285.7 \mathrm{eV}, 287.6 \mathrm{eV}$, and $288.7 \mathrm{eV}$ indicate $\mathrm{sp}^{2}$ hybridized carbon, $\mathrm{C}-\mathrm{O}, \mathrm{C}=\mathrm{O}$, and $\mathrm{COOH}$ groups, respectively $[38,39]$. Both FTIR and XPS data demonstrate that graphite is strongly oxidized by fuming nitic acid and sodium chlorate. In addition, the energy-dispersive X-ray analysis (EDAX) showed a similar result that a certain amount of oxygen was found (as shown in Fig. S1). The Raman spectrum clearly depicted a strong D band at $1339 \mathrm{~cm}^{-1}$ which means defects/disorder-induced mode. The G peak is connected to the in-plane stretching tangential mode of $\mathrm{sp}^{2}$ bonds and was found at $1584 \mathrm{~cm}^{-1}$. This is because the $G$ band derives from the graphene structure, but the $\mathrm{D}$ band bases on defects on graphene [40], which represents that graphite is successfully oxidized which is associated to the FTIR, XPS, and EDAX data. Figure 1(d) exhibits the zeta potential of the GO solution which was around $-55 \mathrm{mV}$, which reveals good distribution of GO in aqueous solution with oxygen-containing groups. This result is constant to the previous FTIR and XPS data. TGA was employed to achieve thermal stability of GO (as shown in Figure 1(e)); a small weight loss was observed before $200^{\circ} \mathrm{C}$, which indicates the evaporation of water absorbed by the hydrophilic group on the GO surface, and the major weight was lost between 200 and $300^{\circ} \mathrm{C}$, exhibiting carbon monoxide, carbon dioxide, and steam because of decomposition of oxygen-related bonds [41, 42], which also depicts that GO has a high oxidation degree.

As shown in Figure 1(f), an evident peak at $2 \Theta=14.15^{\circ}$ was clearly found, which corresponds to a $d$-spacing of $0.63 \mathrm{~nm}$, which depicts that interlamellar water is grabbed between GO layers [43]. Figure 1(g) shows a typical TEM image of graphene nanosheets, and the size of GO was around several hundred nanometers, which is similar to our previous data [35]. These data clearly determine the GO achieved by the modified Brodie method in nanosheets with a sub-micro size.

3.2. Mechanical Property of Gels. Previous literature showed that DN hydrogels owned a better mechanical performance than did PAm single network (SN) hydrogels [8]. To effectively enhance the mechanical performance of DN hydrogels, GO was employed as a nanofiller. In addition, with the help of calcium ions, a calcium ion-induced GO network is built to consume dissipation energy by breaking the network of ionic crosslinks progressively [32]. As shown in Figure 2, TN gels exhibited extraordinary mechanical properties. The hydrogels with high toughness endure large deformations including stretching (Figure 2(a)), knotting (Figure 2(b)), compression (Figure 2(c)), and elongation (Figure 2(d)) without any detectable destruction. TN gels own superior shape recovery rapidly, which makes them quickly recover to the original state after releasing the load. Due to versatile formation, TN gels are widely applied to build various complicated structures, like artificial cartilage. As shown in Figure 3(a), after soaking in $\mathrm{CaCl}_{2}$ solution for 2 hours, the geometry of TN2 gel-based meniscus was retained. 

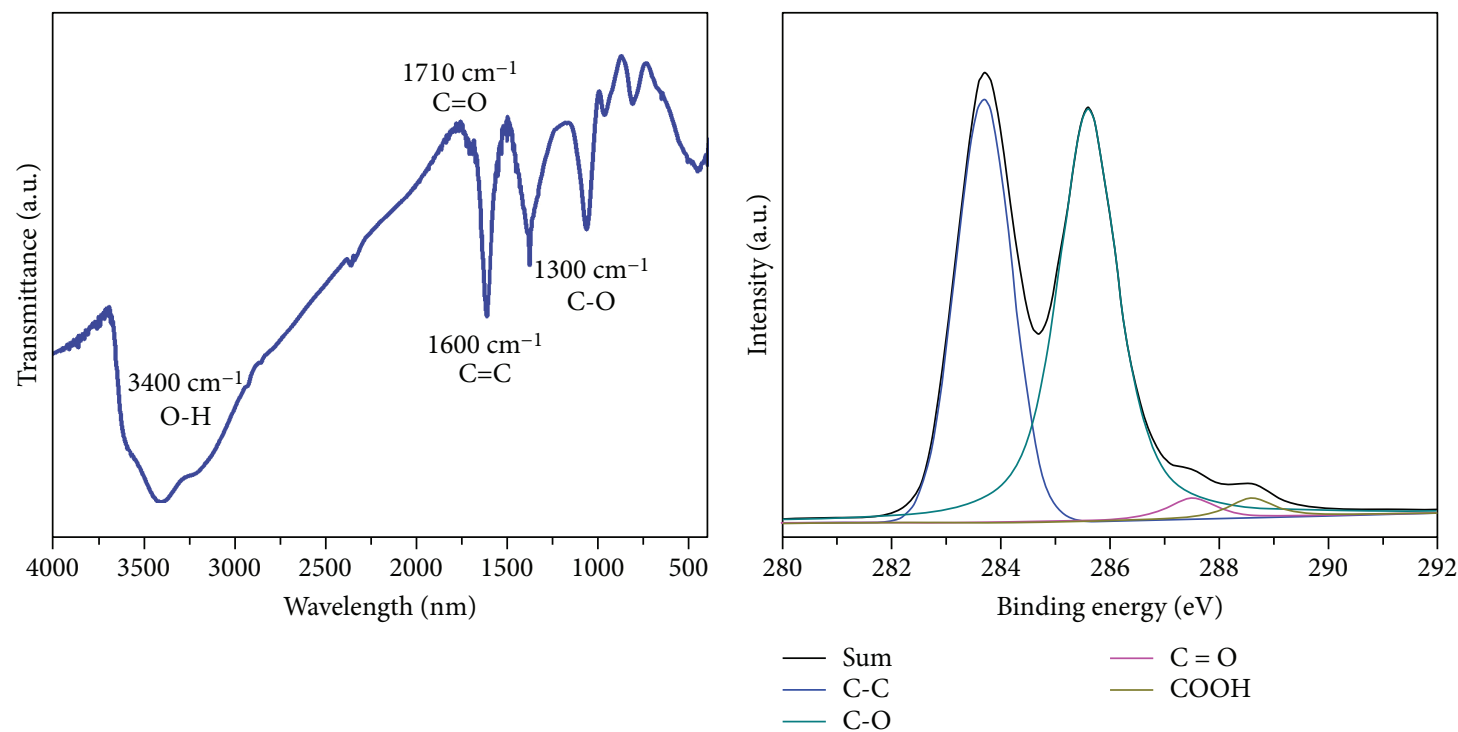

(a)

(b)
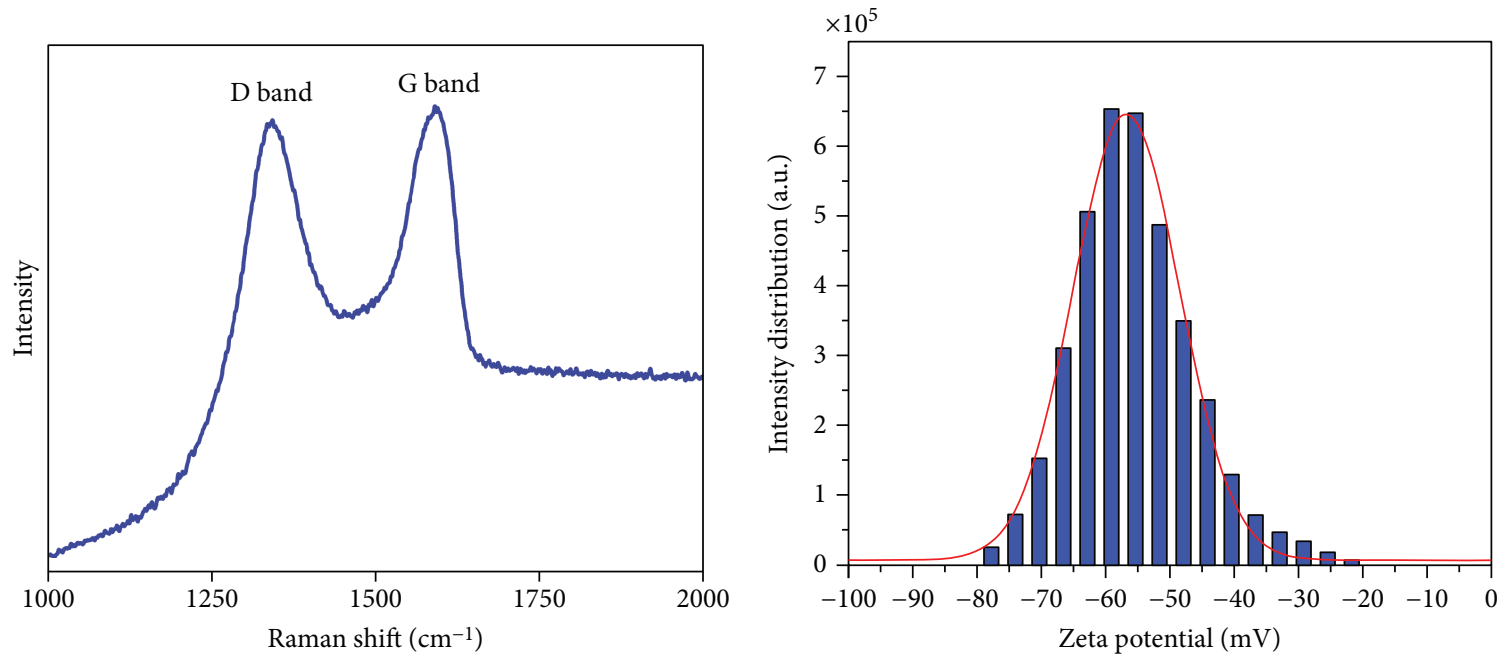

(c)

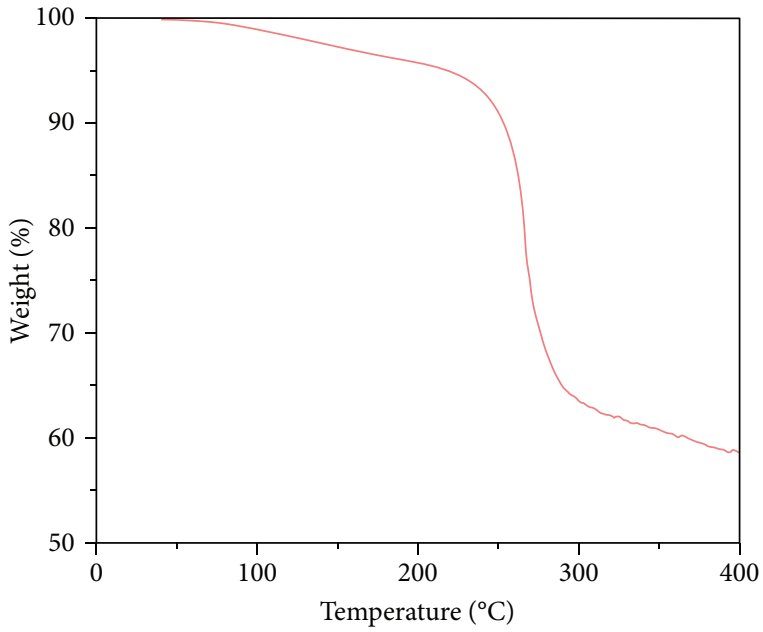

(e)

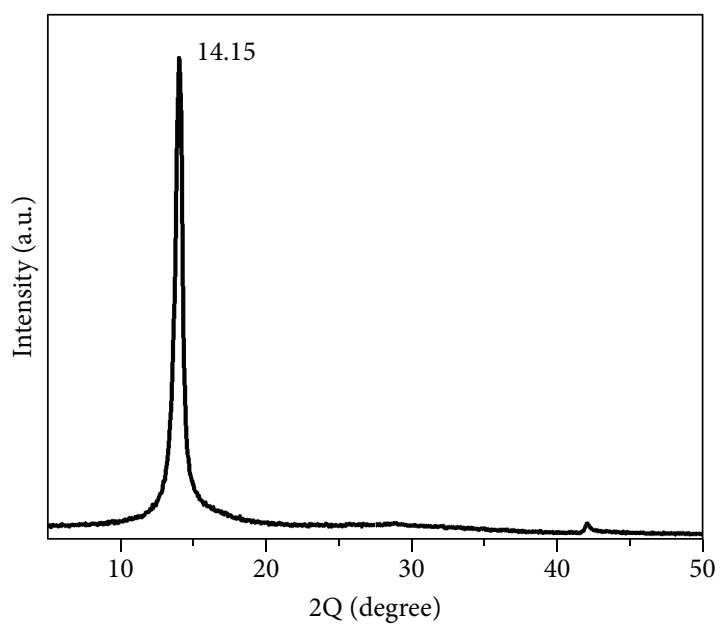

(f)

FIgURe 1: Continued. 


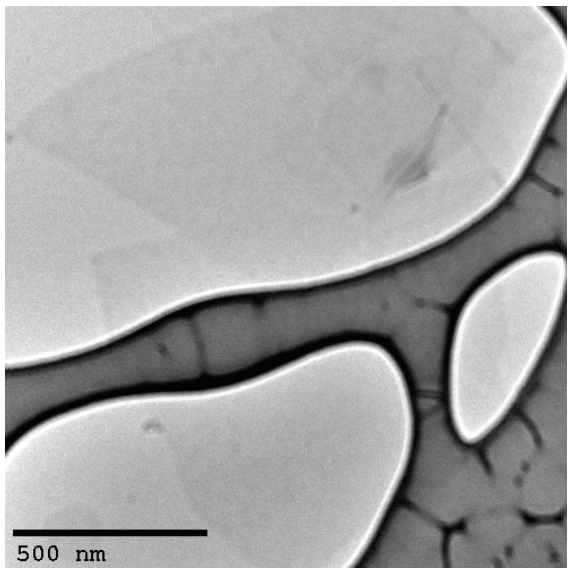

(g)

FIGURE 1: (a) FTIR spectrum of GO, (b) the C1s XPS spectrum of GO, (c) Raman spectrum of GO, (d) the zeta potential distribution of GO aqueous solution, (e) the TGA plot of GO, (f) the XRD pattern of GO, and (g) the TEM image of GO.

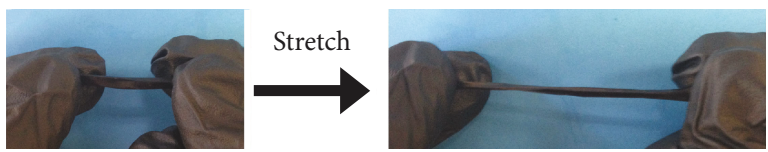

(a)
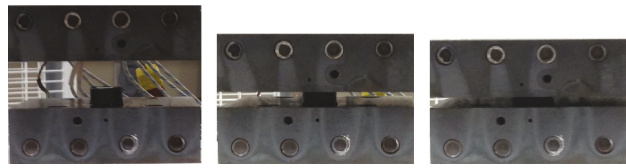

(c)

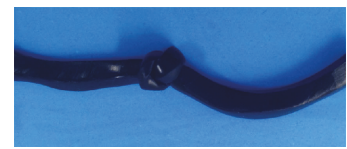

(b)

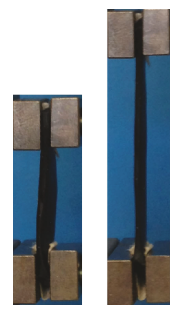

(d)

Figure 2: Excellent mechanical property of TN2 gel, (a) stretching, (b) knotting, (c) compression, and (d) tensile.

Figure 3(b) depicts that TN2 gel-based meniscus possessed excellent geometric consistency in the tibia joint simulator. In addition, this TN2 gel-based meniscus revealed good resiliency and good shape-recovery ability with no noticeable deterioration after twisting and bending (Figures 3(c) and $3(\mathrm{~d}))$.

Unconfined compression tests of $\mathrm{DN}$ and $\mathrm{TN}$ gels were systematically performed, which clearly demonstrated that TN gels achieved superior compressive strength and toughness beyond those for DN gels. The compressive data of the $\mathrm{DN}$ and TN hydrogels with various contents are presented in Figure 4 and Table 2. The DN1 gels (1.06 MPa) showed improved normal stress than did SN gels $(0.37 \mathrm{MPa})$ at $90 \%$ compressive strain. This phenomenon derives from the mechanism that calcium ionically crosslinked alginate consumes dissipation energy and covalently crosslinked PAm preserves elasticity during loading, simultaneously [19]. However, it is still much lower than some reported functional hydrogels in the previous literatures [44]. To effectively increase the mechanical properties of DN1 gels, GO was introduced into the DN1 gels as a reinforced filler. After adding GO, the compressive strength of TN1 (1.46 MPa) and TN2 (2.88 MPa) was largely enhanced, respectively (Figure 4(a)). Similarly, true stress had the analogous tendency. The increased compressive strength results from the entanglement of GO nanosheets with calcium alginate and PAm polymeric networks via physical adsorption, ionic bonding, and hydrogen bonding, which increased the crosslinking and improved both compressive strength and modulus. In addition, the calcium-GO rigid structure acts on absorbing dissipation energy by cracking ionic bonds continuously [32]. In Figure 4(b), TN2 showed a higher normal stress compared to that of TN4 $(2.37 \mathrm{MPa})$ because of increasing alginate amount, which is identical to our previous results [8]. But the excessive amount of alginate could lead to a reduction of compressive strength. Similarly, the compressive strength of DN2 is $0.67 \pm 0.08 \mathrm{MPa}$ which is also smaller than that of DN1. The inferior compressive normal stress derives from that the thick alginate solution restrains the distribution of Am, MBAA, and APS leading to the inhomogeneous and partially polymerized PAm network. The result is consistent to that of swelling properties. It also explains the 


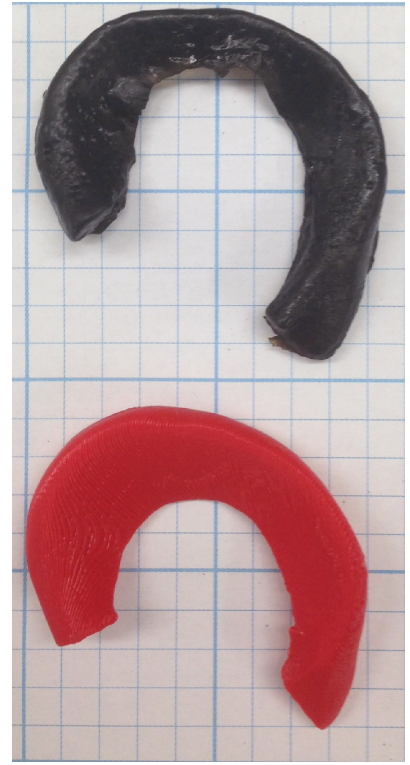

(a)

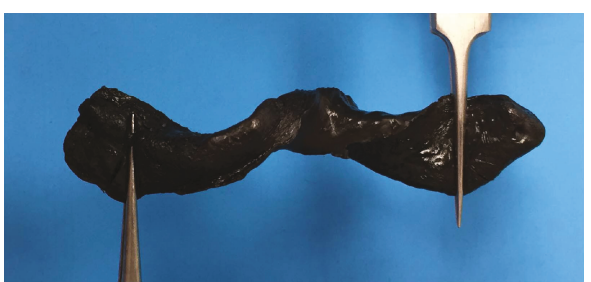

(c)

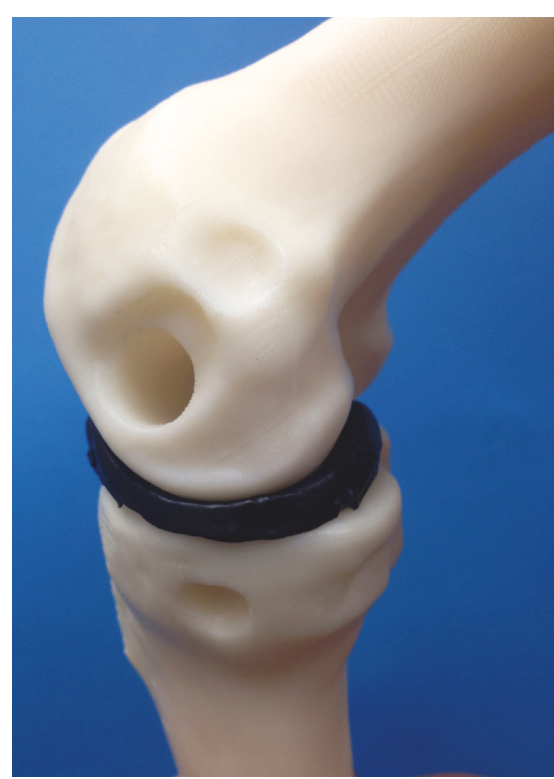

(b)

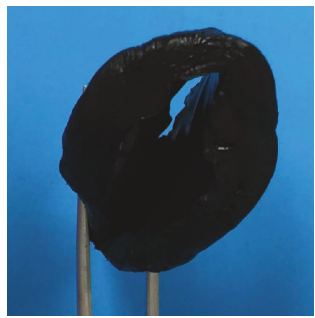

(d)

FIGURE 3: (a) Comparison of the TN2 gel-based artificial cartilage (upper) and the thermoplastic acrylonitrile butadiene styrene- (ABS-) based cartilage geometric model (bottom, dyed in red). (b) The photographs of the gel-based cartilage with good geometric consistency with the tibia joint simulator. (c) Twisting of gel-based cartilage. (d) Bending of gel-based cartilage.

low swelling ratio of DN2 and TN3 gels (Fig. S2). The PAm network contributes the most swelling property of DN and TN gels, and the low polymerization of PAm generates the low swelling ability of gels.

Cyclic loading-unloading measurements were performed to study fatigue resistance of DN and TN gels. Figure 5(a) presents that the adjacent loading curves were overlapped, which is distinctive among conventional double network hydrogels including agar/PAm [11] and poly(2-acrylamido-2-methylpropanesulfonic acid) (PAMPS)/PAm DN gels [45]. Although these gels show superior mechanical properties in the first cycle, the brittle nature of the first network results in mechanical failure after deformation. The corresponding cyclic loading-unloading curves present that the large strain loading curves and previous unloading curves are overlapped when the strain is smaller than the maximum strain, and the further deformation leads to a fast enhancement in stress because of the fracture of the residual sacrificial network. Even with the help of chondroitin sulfate-coated vinyl silica nanoparticles (CS-SNP) and poly(3,4-ethylenedioxythiophene) (PEDOT) belt mesh, the following loading curve has a larger strain deviation than the preceding unloading curve $[11,45]$. Therefore, the calcium alginate/PAm DN gels have better mechanical recoverability than other nonionically crosslinked double network gels. As shown in Figure 5(b), an identical phenomenon was achieved, which indicates that the calcium-induced GO network is also reversible after deformation. The TN2 gels also showed a higher loading curve than did DN1 gels, which demonstrates that the electrostatic interaction between the GO and polymeric chains and the calcium ion-induced GO network results in energy consumption.

The systematic investigation was performed via periodic loading-unloading measurements with a certain strain. Figures 5(c) and 5(d) present that TN2 gels showed a bigger hysteresis loop than did DN1 gels, and both hysteresis loops become smaller in subsequent loading, which indicates that the calcium crosslinking of the alginate network is only partially recovered not fully. The difference among subsequent loading cycles in DN1 gels was slight, because of the break in the calcium alginate network that cannot be rebuilt in short time. The compressive stress ( $90 \%$ strain) of DN1 gels negligibly decayed from $1.39 \mathrm{MPa}$ for the first loading to 1.31 MPa, 1.28 MPa, 1.29 MPa, and 1.31 MPa for the 2nd, 3 rd, 4th, and 5th run (Figure 5(c) inset). The DN1 gels have abilities to retain the most compressive property (around $92 \%)$ at $90 \%$ strain. However, among the subsequent loading cycles in TN2 gel (Figure 5(d) insert), a visible decline was 


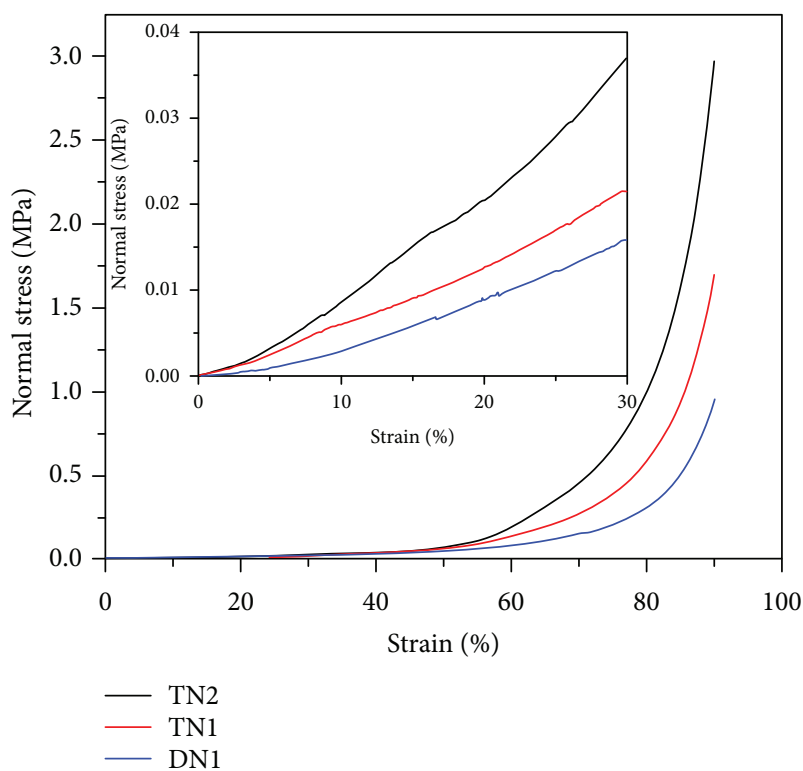

(a)

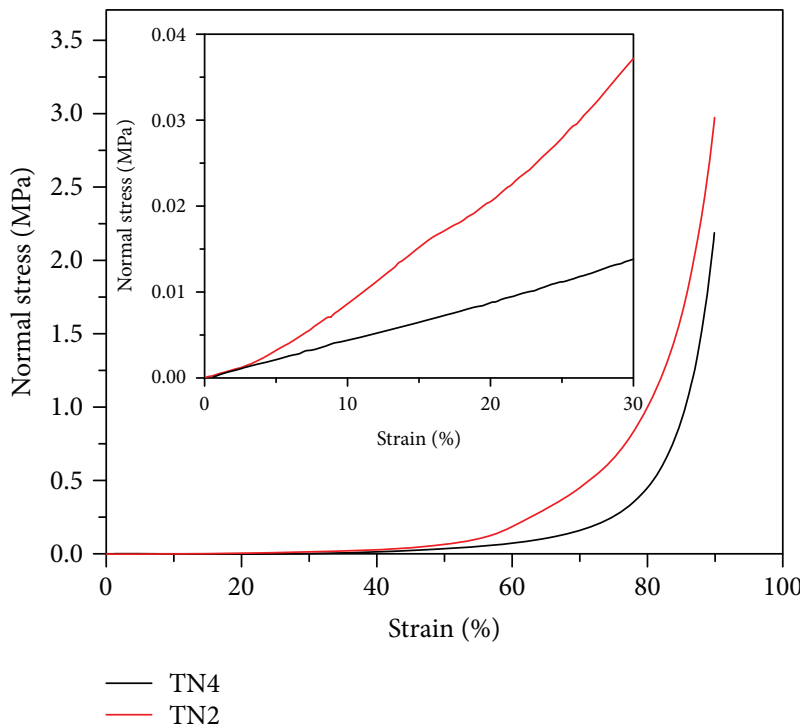

(c)

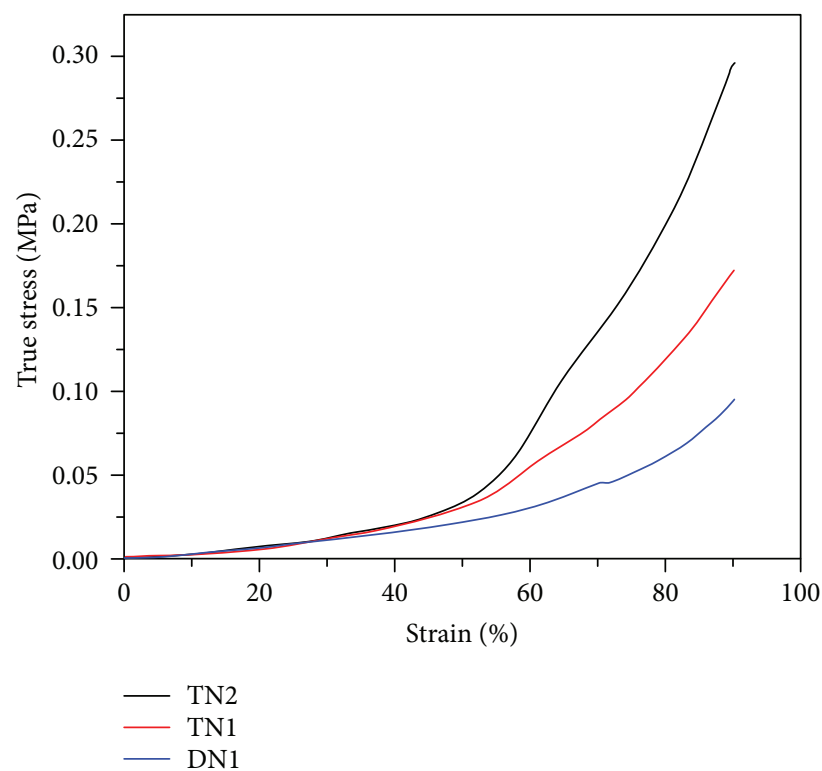

(b)

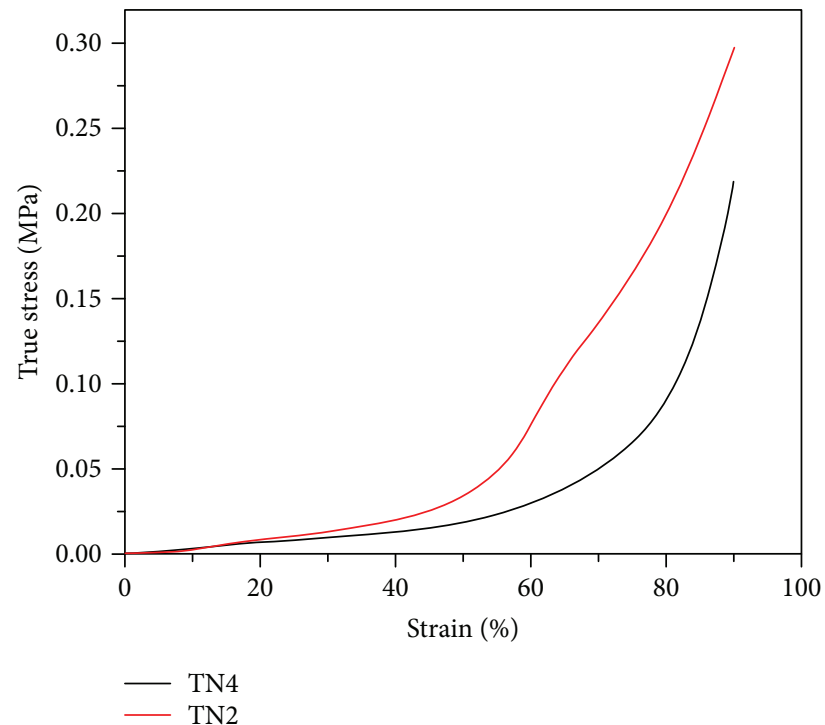

(d)

FIgURE 4: Normal stress-strain curves of gels with different GO amounts (a) and different alginate amounts (c). True stress-strain curves of gels with different $\mathrm{GO}$ amounts (b) and different alginate amounts (d).

TABLE 2: The compressive mechanical properties of hydrogels with different compositions.

\begin{tabular}{lcc}
\hline Hydrogels & Normal strength $(\mathrm{MPa})$ & Toughness $\left(\mathrm{kJ} \mathrm{m}^{-3}\right)$ \\
\hline SN & $0.37 \pm 0.03$ & $86.93 \pm 0.97$ \\
DN1 & $1.06 \pm 0.13$ & $102.71 \pm 2.81$ \\
DN2 & $0.63 \pm 0.02$ & $105.88 \pm 12.90$ \\
TN1 & $1.46 \pm 0.20$ & $142.81 \pm 27.71$ \\
TN2 & $2.88 \pm 0.18$ & $281.77 \pm 19.58$ \\
TN3 & $2.02 \pm 0.67$ & $354.11 \pm 90.41$ \\
TN4 & $2.37 \pm 0.17$ & $177.54 \pm 25.90$ \\
\hline
\end{tabular}

observed. This is because the displacement of GO nanosheets under large deformation damages the calcium ion-induced GO network progressively resulting in property decay. The stress (90\% strain) of TN2 gels decreases from $2.39 \mathrm{MPa}$ (first loading) to $2.08 \mathrm{MPa}, 1.98 \mathrm{MPa}, 1.93 \mathrm{MPa}$, and $1.92 \mathrm{MPa}$ for the second, third, fourth, and fifth cycles, respectively. This demonstrates that the calcium ion-induced GO network has limited reversible ability due to displacement of GO nanosheets in polymeric networks that is hard to be fully recovered. The dissipation energy was also calculated according to the loading-unloading curves. As shown in Figure 5(e), the dissipation energy decreased with increasing cycles. However, after five cycles, the dissipation energy of TN2 gels was kept at around $110 \mathrm{~kJ} \mathrm{~m}^{-3}$ which is close to that of animal cartilage (around $100 \mathrm{~kJ} \mathrm{~m}^{-3}$ ) [46]. This indicates that these 


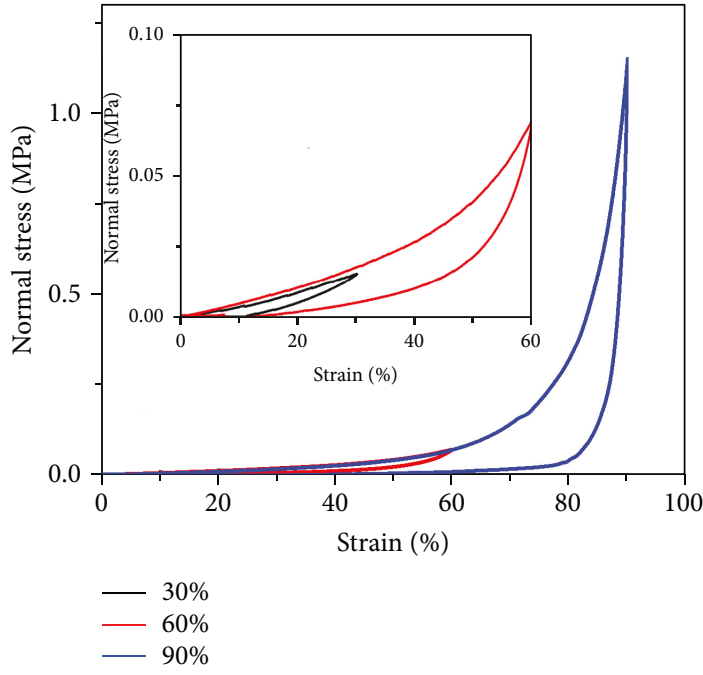

(a)
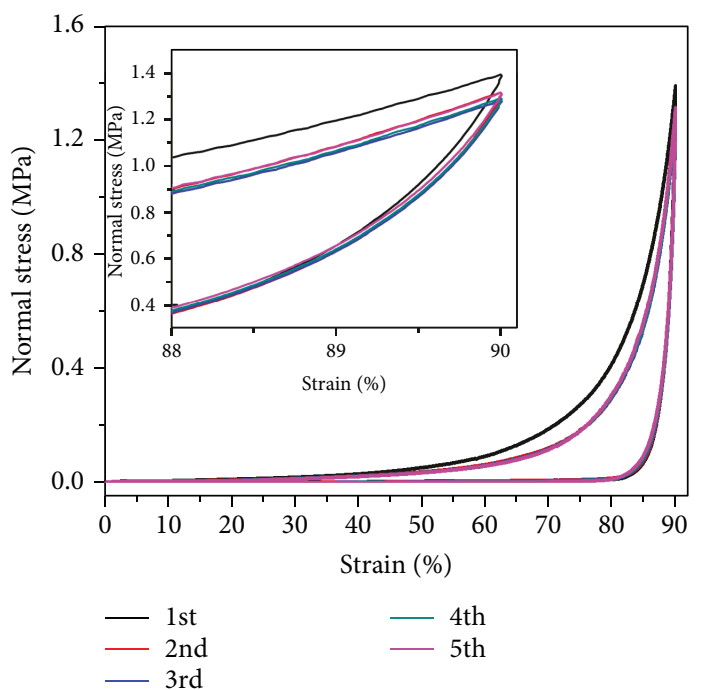

(c)

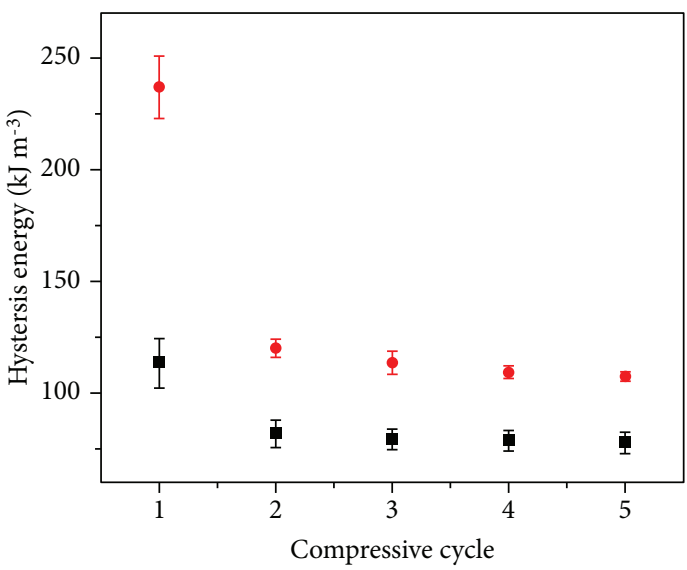

- DN1

- TN2

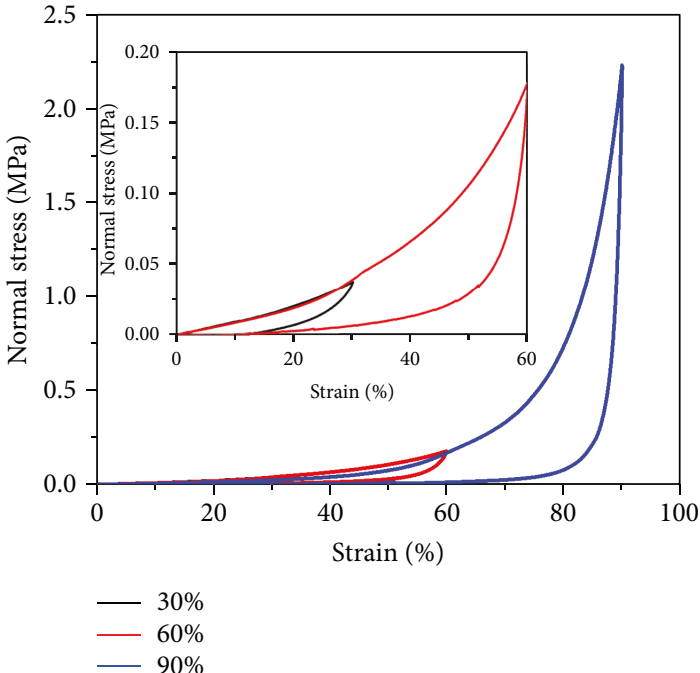

(b)
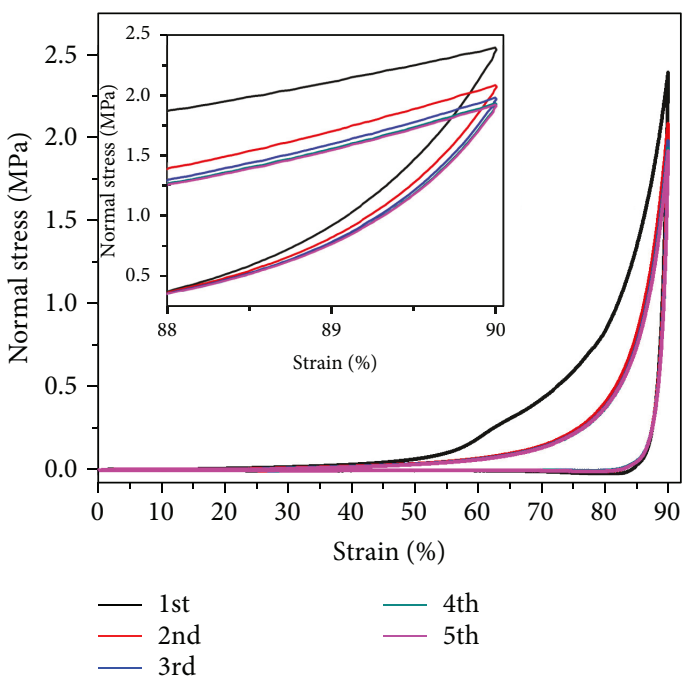

(d)

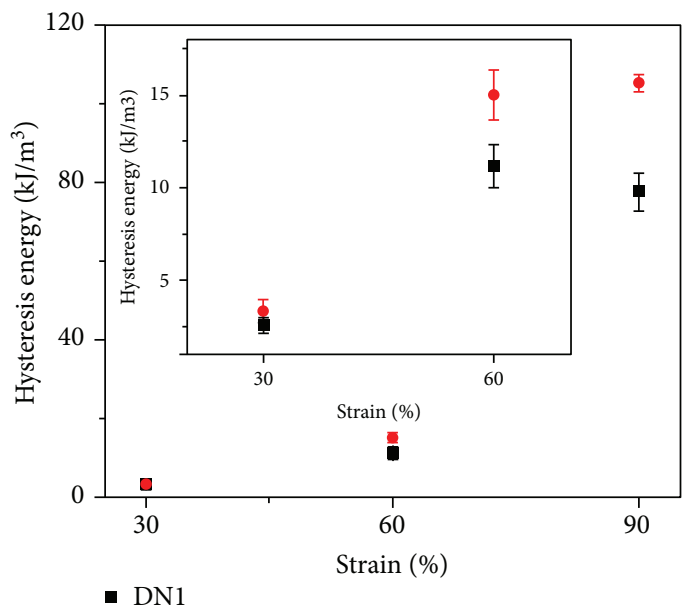

- TN2

(e)

Figure 5: Continued. 


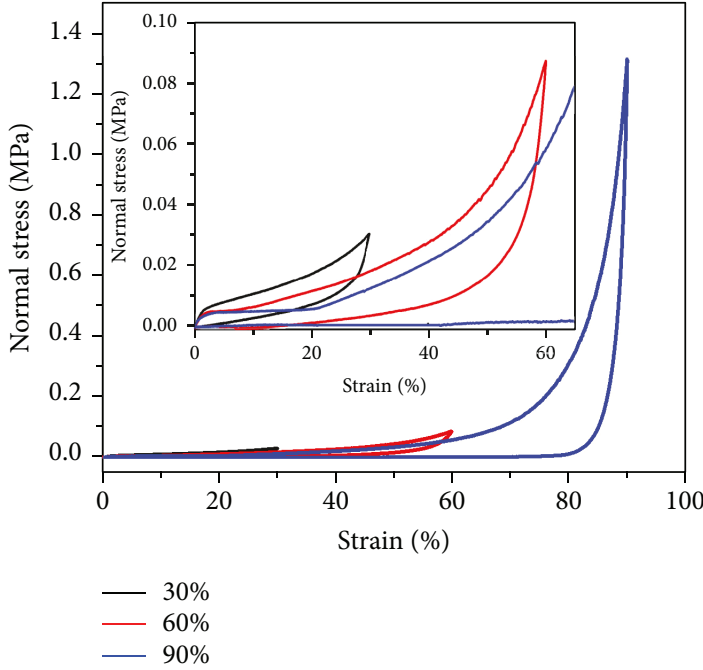

(g)

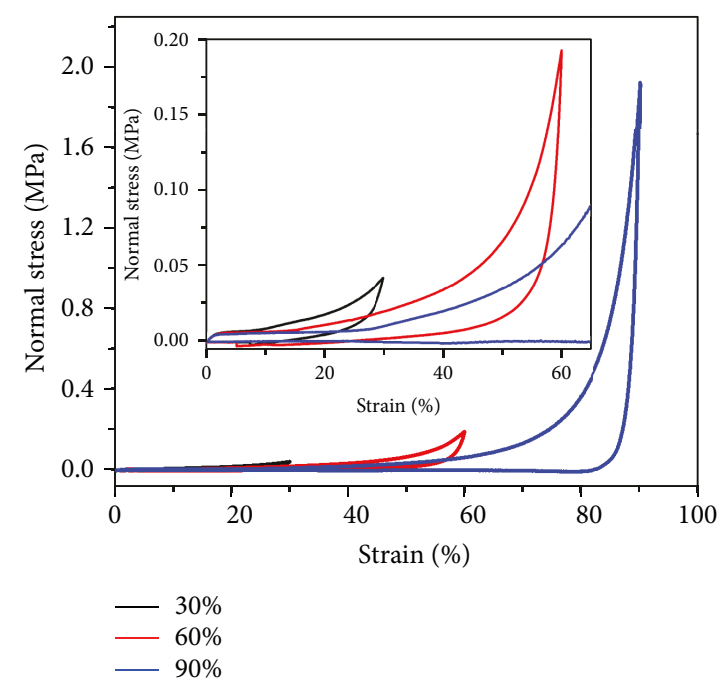

(h)

FIGURE 5: The typical consecutive loading-unloading curves with gradient increased in maximum strain on (a) DN1 and (b) TN2 in as-prepared state. Representative cyclic loading-unloading curves of (c) DN1 and (d) TN2 hydrogels for up to five cycles. (e) The dissipation energies decay with increasing loading cycles at $90 \%$ maximum strain, and (f) the dissipation energies of the fifth cycle with increasing strain. The hysteresis loops at the fifth loading cycle at different maximum strains of (g) DN1 and (h) TN2.

TN2 gels can be employed as a promising substitute in load-bearing tissues. Cyclic compressive measurements were also tested at other strains (30\% and 60\%). With increasing test number, the compressive stress and loop area showed a small decay (Fig. S3). Figures 5(g) and 5(h) present the fifth loop at three various strains $(30 \%, 60 \%$, and $90 \%)$. The dissipation energy values were largely improved with increasing strain. The dissipation energy values of the fifth cycle with different strains are summarized in Figure 5(f), which presented that the dissipation energy was exponentially improved with enhanced strain. It indicates that both DN1 and TN2 gels achieve a cyclic-independent self-toughing performance of these synergistic networks when the strain is at a high level, compared to other double network gels like agar/PAAm DN gels [10]. This is an essential property to be a candidate material for replacement of load-bearing tissues, which is derived from the fluid pressurization which is a critical role in cartilages.

3.3. Swelling Property of Gels. To comprehensively investigate the swelling behavior of DN and TN gels, the results including size change and ESD were performed. As shown in Figure 6(a), the diameter of cylindrical gels rapidly increased in the first 24 hours, which depicts favorable ability of hydrogels to swell. When hydrogels in their original state soaked in water solution, the water molecules attack the gel's surface and invade into the hydrogels leading to an extension of polymeric networks in the rubbery phase [47]. After 48 hours, the diameters were stable without large increase. The inset images also showed that the sizes of swelling gels ( 2 days, 9 days, and 11 days) were nearly changed; however, an obvious change was found among swelling gels after soaking in DI water 0 hour, 4 hours, 8 hours, and 1 day, respectively. These results depict that swelling of hydrogels is not a continuous process and the swelling process stops when the elastic and osmotic forces are balanced at the swelling equilibrium. The changes in diameter have consistent tendency, although the diameter of DN1 cylindrical gels (around $21.4 \mathrm{~mm}$ ) is much larger than that of TN2 cylindrical gels (around $19.7 \mathrm{~mm}$ ). That means the presence of GO restrains the swollen property of gels via physical crosslinking that long polymeric chains interpenetrate the tough nanofillers and the chemical bonding between oxygen-containing groups on GO surfaces and hydroxyl, carboxyl, and amine groups on polymeric chains. Differently, the diameter of SN gels was still continuously increased after 2 days and was around $26 \mathrm{~mm}$ at 8 days of soaking. In addition, the gels were so soft to hold their original shape and were easy to be broken after handling with long-time soaking (as shown in Fig. S4). This is because hydrogels are considered as an arbitrary dispersion of crosslinkings on a lattice built by the interchain contact points [48]. After soaking in solvent, the small polymeric clusters became larger ones, creating regions with low concentration resulting in mechanical failure. The fully swollen sizes of gels with different compositions are shown in Figure 6(b). With addition of calcium alginate, the hard calcium alginate network not only works as a tough frame in DN1 gels and binds to the soft PAm network by chemical bonding. With increasing amount of alginate, the swollen sizes of DN1 gels decline which derives from the low swelling property of alginate hydrogels that the calcium alginate network suppressed the swelling of PAm hydrogels [8]. On the other hand, with addition of GO, the fully swollen size of TN2 gels was much smaller, compared to that of DN1 gels, which indicates that the addition of nanofillers into DN2 hydrogels can largely restrict the swollen ability of hydrogels due to the physical interpenetrations and chemical bindings. With increasing amount of GO in TN gel, the diameter of swelling gels was closer to that of as-prepared gels, which demonstrates that the more GO leading to more contacts in hydrogels decreases 


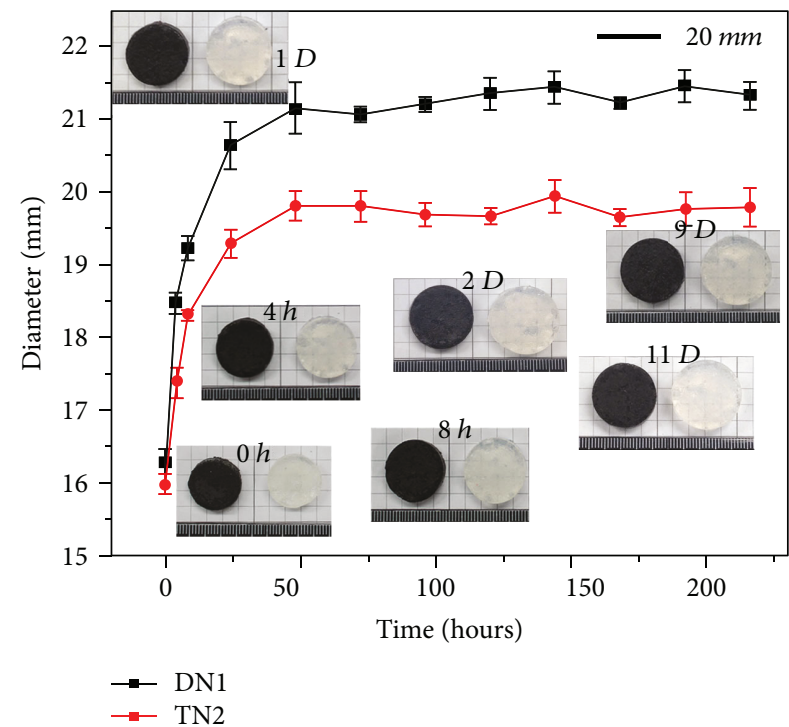

(a)

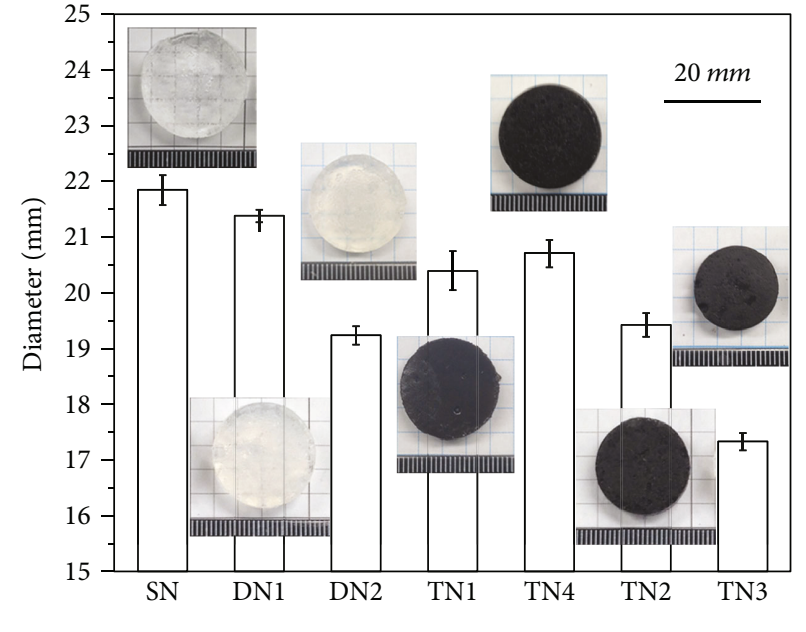

(b)

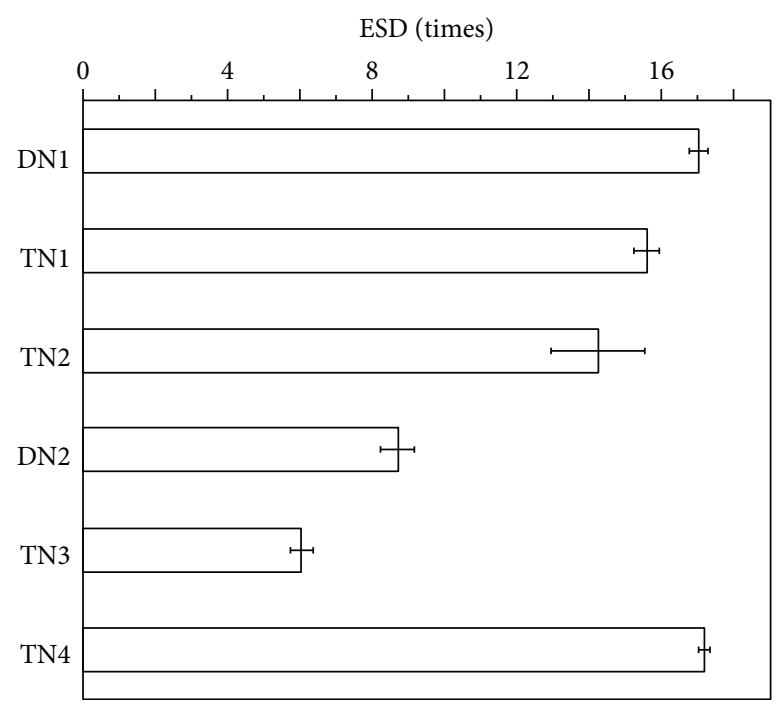

(c)

Figure 6: (a) Size of the DN1 and TN2 gels, (b) final shape and size in swollen state (the data of SN is achieved after soaking in DI water for 2 days), and (c) ESD of hydrogels.

the swollen property of DN gels. In addition, the ESD was also calculated and is depicted in Figure 6(c). With inclusion of GO and calcium alginate, less water content was contained in fully swelling hydrogels, which is consistent with above results.

As shown in Scheme 1, a systematic and schematic figure was presented to explain the two-step fabrication process and swelling change of DN and TN gels. In the first step, the PAm SN was covalently crosslinked by MBAA and the cylindrical gels were formed with mold geometry under UV light exposure (Scheme 1(a)). On the other side, as presented in Scheme 1(b), GO nanosheets were intertwined in PAm SN via physical interpenetrations, ionic bonding, and hydrogen bonding as physical crosslinks [29]. In the second step, the gels were soaked in the $8 \mathrm{wt} \% \mathrm{CaCl}_{2}$ solution to build a calcium alginate network and a calcium-induced GO network. The sodium alginate chains were ionically bonded to each other with introduction of calcium ions (Scheme 1(c)), whereas, in TN2 gels (Scheme 1(d)), besides calcium alginate polymeric network, an ion-induced GO network was also formed via hydrogen bonding between GO and calcium ions [32]. In addition, intertwined bonds were built between amine groups on PAm and carboxyl groups on alginate in both DN and TN gels [15]. The size of DN1 was increased from $15.83 \pm 0.04 \mathrm{~mm}$ to $16.27 \pm 0.18 \mathrm{~mm}$ (Scheme 1(c)), which is similar to our previous data [8], which indicates that the formation of the calcium alginate network temporarily suppresses the swelling property of gels. With the help of a binding force derived from the crosslinking process between calcium ions and $\mathrm{G}$ units in alginate polymeric chains, the hydrogels did not depict regular volumetric swelling, whereas the size of TN2 was increased to $15.97 \pm 0.13 \mathrm{~mm}$ (Scheme $1(\mathrm{~d})$ ), which was much closer to their original size. 


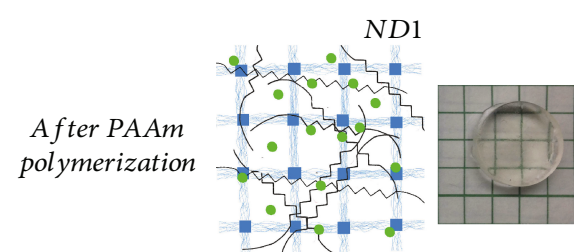

(a)

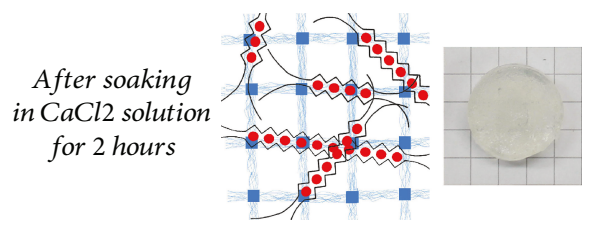

(c)

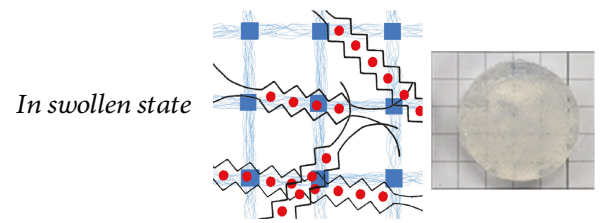

(e)

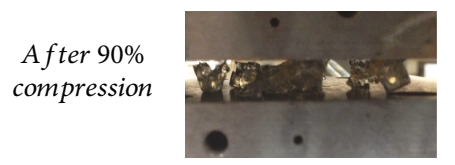

(g)

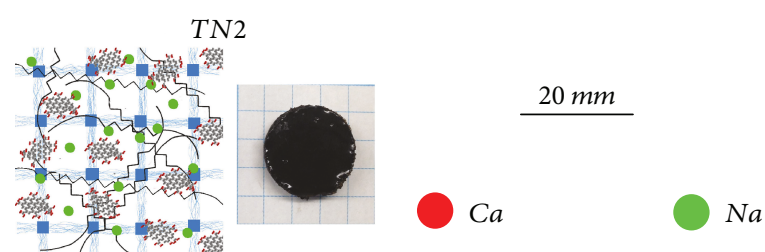

(b)
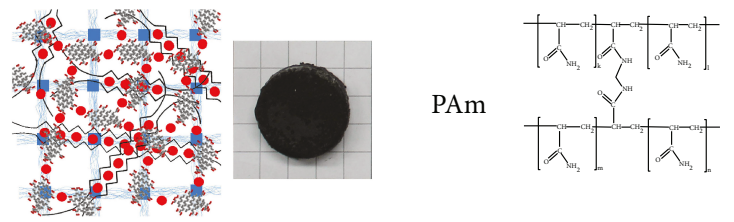

(d)

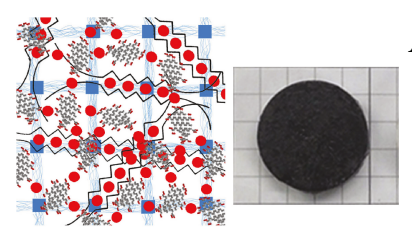

(f)
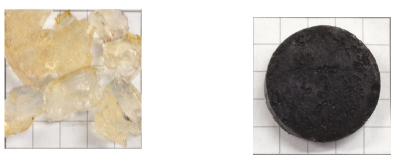

GO

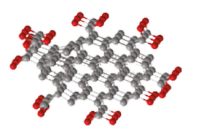

(h)

Scheme 1: The diagram of DN and TN gels with photos. After polymerization (a) DN1, (b) TN2, after soaking in CaCl ${ }_{2}$ solution for 2 hours, (c) DN1, (d) TN2, in swollen state, (e) DN1, (f) TN2, after 90\% compression, (g) DN1, and (h) TN2.

The reason is that the GO sheets integrate with the polymer chain and keep the elastic energy of polymeric chains, which suppress the solvent permeation via osmotic energy. After being fully swollen in DI water (Schemes 1(e) and 1(f)), the solvent is introduced into the gaps between polymeric networks via osmotic pressure to broaden capacities among covalent crosslinked and ionic crosslinked points. Compared to DN (Scheme 1(e)) and SN gels (Fig. S5), the TN hydrogels allow less solvent leak into the gels (Scheme 1(f)), due to more crosslinked points and more bonds between GO and the polymeric network. At the swelling equilibrium, the size of DN1 was increased to $21.37 \pm 0.11 \mathrm{~mm}$; however, the size of TN2 was $19.41 \pm$ $0.21 \mathrm{~mm}$, which demonstrates that the physical and chemical contacts derived from GO rigid nanosheets can greatly suppress the swelling nature of DN gels. Surprisingly, Scheme $1(\mathrm{~g})$ shows that the DN1 swollen gels were crashed into small parts after large strain compression; however, TN2 swollen gels presented the ability to recover its shape, which is depicted in Scheme 1(h). The swelling strongly damages the elastic behavior of hydrogels via introduction of water molecules into clusters and stretching the polymeric network. However, with the addition of the calcium alginate network and the chemical binding between carboxyl groups on alginate and amine groups on PAm [15], more elastic energy is preserved in the swelling equilibrium, which makes the calcium alginate/PAm DN hydrogels have the ability to endure a certain degree of

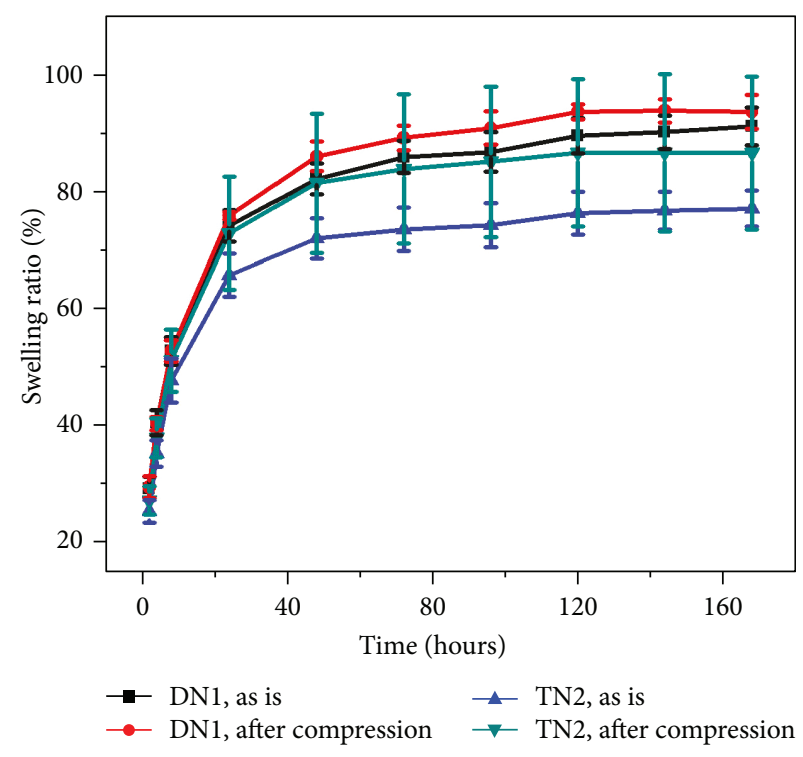

FIgURE 7: Reswelling of DN and TN hydrogels with or without compression test at a strain of $90 \%$.

fracture energy not like PAm SN hydrogels that possessed a soft nature in swelling state. With addition of GO in DN gels, the TN gels showed superior mechanical property even in swollen state. The GO nanosheets not only 


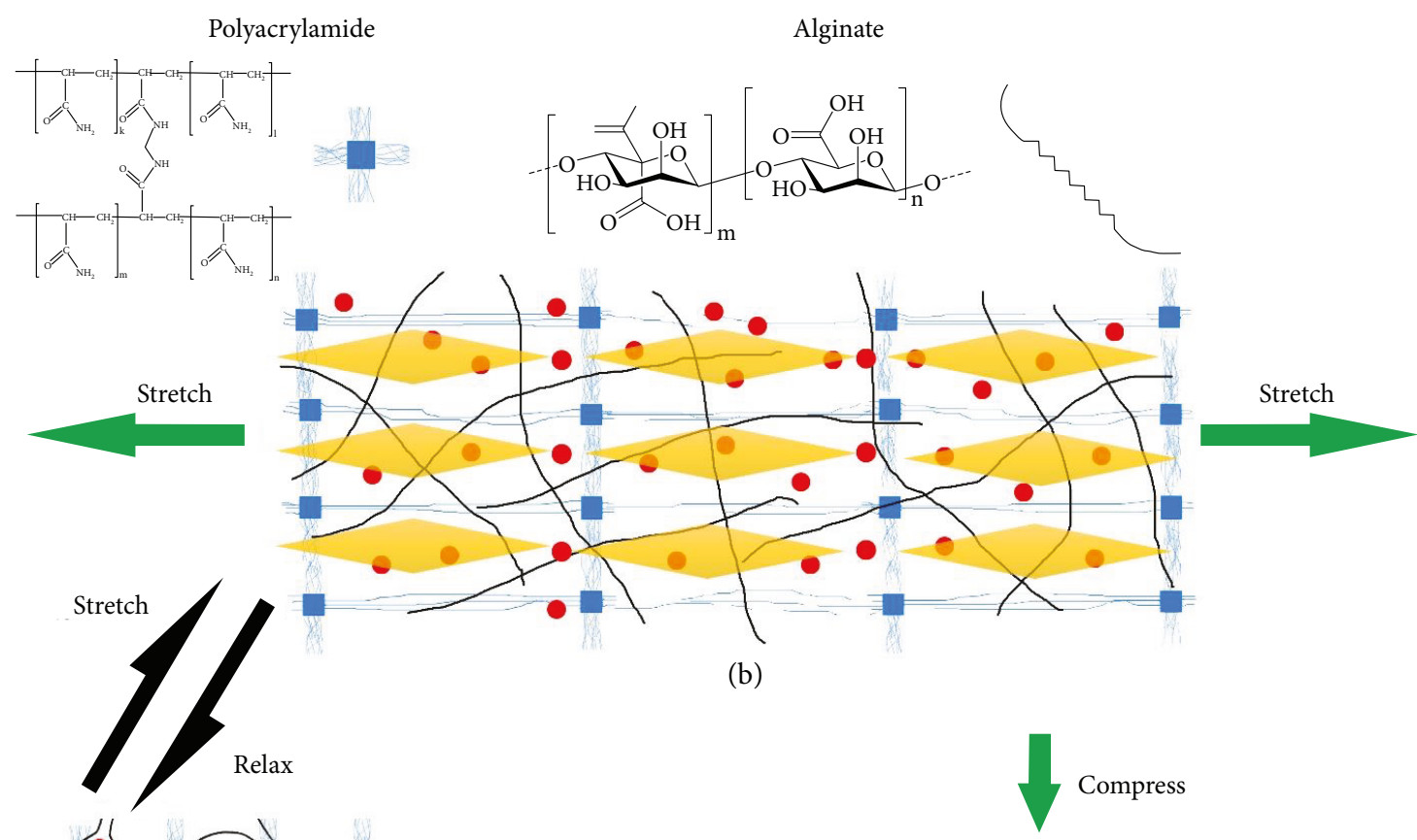

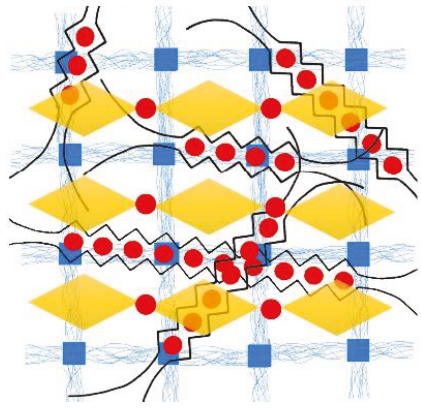

(a)

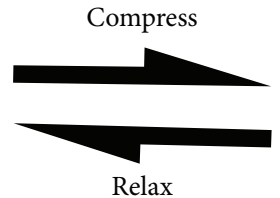

Relax

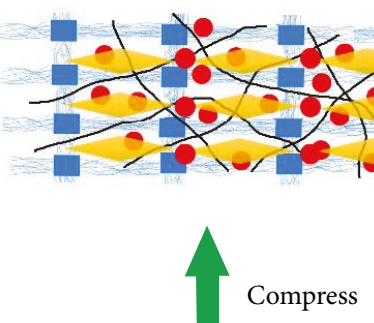

(c)

Calcium ion

Graphene oxide

Scheme 2: Schematic models of the TN hydrogels. (a) Types of crosslinking networks in the TN hydrogels including an ionically crosslinked alginate network, calcium ion-induced GO physical network, and covalently crosslinked PAm network; (b) stretch model for the TN hydrogels; (c) compression model for the TN hydrogels.

suppress the swelling nature of gels via physical interpenetrations and chemical bindings as additional crosslinks to retain more elastic energy but also dissipates mechanical energy during the large deformation.

The internal fracture and fluid pressurization behavior of these DN1 and TN2 hydrogels were further studied by soaking tests of as-prepared and compression-tested gels. Figure 7 depicts that all hydrogels possessed a substantial increase in the swollen ratio with time until equilibrium. In both $\mathrm{DN}$ and TN hydrogels, the ultralong PAm network affords a high osmotic pressure to introduce water into the DN and TN gels. In addition, the swelling ratio of $\mathrm{DN}$ gels was around $91 \%$, which is higher than that of TN gels (around $77 \%$ ). Due to the presence of calcium-induced GO networks, large numbers of intertwined bonds were formed among functional groups on GO, amine groups on the PAm network, and carboxyl groups on the calcium-alginate network in TN gels [20], which effectively restricted the swelling property of the PAm network. After compression, the swelling ratio of compression-tested DN gels was around 93\%, which is similar with that of as-prepared DN gels. These results indicate that most sacrificial calcium alginate networks are recovered after compression, whereas the swelling ratio of TN gels was slightly increased to $86 \%$, which is still smaller than that of as-prepared DN hydrogels. These results of the swelling ratio exhibit the compression results in a small displacement of GO leading to a minor fracture of the calcium-induced GO network, which corresponds well to the mechanical results of TN gels. The restraining effect of the calcium-induced GO network is similar with that of collagen networks in the cartilage extracellular matrix (ECM) and provides energetic immobilization of imbibed fluid. These TN hydrogels with improved toughness and superior fatigue resistance acquire the potential application in artificial cartilage.

3.4. Triple Network. Based on the above investigation, Scheme 2 depicts a schematic figure of biomimetic TN gels. 
The construction of TN hydrogels is derived from a synergistic effect of four different groups of interactions: calcium ion-induced GO physical networks, calcium ion crosslinking of alginate networks, covalent crosslinking of PAm networks, and hydrogen binding among GO, calcium alginate, and PAm. Three-dimensional GO networks were built by forceful coordinations between oxygen-related groups of GO nanosheets and fragile bindings between calcium ions and oxygen-containing groups on the GO surface [32]. Both alginate network and PAm network were interlaced with nanofillers via hydrogen bonds, leading to a tough network [8]. This unique TN structure of these hydrogels greatly facilitates the improvement of compressive and tensile strength during deformation. Under loading cycles, the covalent crosslinking of PAm maintains elasticity, while the reversible calcium-alginate and calcium-GO networks act on consuming energy by cracking the ionically crosslinked networks. In addition, the chemical bindings among $\mathrm{GO}$, calcium alginate, and PAm preserve the original state of these TN hydrogels. Due to reversible calcium crosslinking of the alginate network and calcium ion-induced GO physical network that can sufficiently release local stress and consume energy, TN hydrogels achieve superior toughness and fatigue resistance. Schemes 2(b) and 2(c) present the simplified models to describe visually the superior toughness and fatigue resistance of these TN hydrogels during compression and stretch, respectively.

\section{Conclusions}

In summary, graphene oxide- (GO-) reinforced triple network (TN) hydrogels have been first developed via introduction of the calcium-induced GO network into calcium alginate/polyacrylamide double network (DN) hydrogels. Compared to conventional DN gels, these TN gels achieve superior toughness and fatigue resistance, simultaneously. Due to the addition of GO, numerous crosslinking bonds are formed among functional groups on GO, amine groups on polyacrylamide (PAm) chains, and carboxyl groups on alginate chains, which enhances the crosslinking degree of the TN hydrogels. The increased crosslinking degree and rigid nature of $\mathrm{GO}$ effectively improve the compressive strength (around 172\%) and toughness (around 174\%) of these TN gels compared to DN hydrogels. Due to the existence of the partially reversible calcium alginate network and calcium-induced GO networks, the TN hydrogels achieve excellent fatigue resistance. Upon five cycles, the toughness of hydrogels remains around $110 \mathrm{~kJ} \mathrm{~m}^{-3}$, which is similar with that of animals' articular cartilage. The swelling results methodically demonstrate that the calcium GO-induced network can effectively restrict the swelling property of the PAm network, which results in superior mechanical maintenance. The internal fracture behavior of these TN hydrogels is studied via the swelling tests of compressed and as-prepared gels. The restraining effect of the calcium-induced GO network offers strong immobilization of imbibed fluid, which is similar with that of the collagen network of the cartilage extracellular matrix. Therefore, these supertough and fatigue-resistant gels may be an auspicious substitute for cartilage.

\section{Data Availability}

The data used to support the findings of this study are included within the article and supplementary information file.

\section{Conflicts of Interest}

The authors declare that there are no conflicts of interest regarding the publication of this paper.

\section{Acknowledgments}

The authors would like to acknowledge the support from National Science Foundation grant \# 1228127. The authors thank the Imaging Centre of Texas Tech University for the TEM and Dr. Golden Kumar for the compression testing machine.

\section{Supplementary Materials}

Figure S1: EDAX spectrum of graphene oxide. Figure S2: swelling ratio of A4P12, G1A2P12, and G1A4P12. Figure S3: representative cyclic loading-unloading curves of $\mathrm{A} 2 \mathrm{P} 12$ hydrogels at (a) 30\% and (b) 60\% strain for up to five cycles, and representative cyclic loading-unloading curves of G1A2P12 hydrogels at (c) $30 \%$ and (d) $60 \%$ strain for up to five cycles. Figure S4: size change of P12 single network hydrogel; the inset is the shape of P12 SN hydrogel after 9-day soaking in DI water. Figure S5: schematic figure of SN gel in swelling state. (Supplementary Materials)

\section{References}

[1] K. Arakaki, N. Kitamura, H. Fujiki et al., "Artificial cartilage made from a novel double-network hydrogel: in vivo effects on the normal cartilage and ex vivo evaluation of the friction property," Journal of Biomedical Materials Research Part A, vol. 93, no. 3, pp. 1160-1168, 2010.

[2] G. A. Ateshian, "Weaving in three dimensions," Nature Materials, vol. 6, no. 2, pp. 89-90, 2007.

[3] L. E. Freed, G. C. Engelmayr Jr., J. T. Borenstein, F. T. Moutos, and F. Guilak, "Advanced material strategies for tissue engineering scaffolds," Advanced Materials, vol. 21, no. 32-33, pp. 3410-3418, 2009.

[4] G. C. Engelmayr, M. Cheng, C. J. Bettinger, J. T. Borenstein, R. Langer, and L. E. Freed, "Accordion-like honeycombs for tissue engineering of cardiac anisotropy," Nature Materials, vol. 7, no. 12, pp. 1003-1010, 2008.

[5] A. Fukuda, K. Kato, M. Hasegawa et al., "Enhanced repair of large osteochondral defects using a combination of artificial cartilage and basic fibroblast growth factor," Biomaterials, vol. 26, no. 20, pp. 4301-4308, 2005.

[6] J. R. Steadman, W. G. Rodkey, K. K. Briggs, and J. J. Rodrigo, "The microfracture technique to treat full thickness articular cartilage defects of the knee," Der Orthopäde, vol. 28, no. 1, pp. 26-32, 1999. 
[7] M. Ochi, Y. Sumen, J. Jitsuiki, and Y. Ikuta, “Allogeneic deep frozen meniscal graft for repair of osteochondral defects in the knee joint," Archives of Orthopaedic and Trauma Surgery, vol. 114, no. 5, pp. 260-266, 1995.

[8] J. Wang, J. Wei, S. Su, J. Qiu, and S. Wang, "Ion-linked double-network hydrogel with high toughness and stiffness," Journal of Materials Science, vol. 50, no. 16, pp. 5458-5465, 2015.

[9] J. Wei, J. Wang, S. Su, M. Hasan, J. Qiu, and S. Wang, “A shape healable tough hydrogel," New Journal of Chemistry, vol. 39, no. 11, pp. 8461-8466, 2015.

[10] Q. Chen, L. Zhu, C. Zhao, Q. Wang, and J. Zheng, "A robust, one-pot synthesis of highly mechanical and recoverable double network hydrogels using thermoreversible sol-gel polysaccharide," Advanced Materials, vol. 25, no. 30, pp. 4171-4176, 2013.

[11] J. Wei, J. Wang, S. Su, S. Wang, and J. Qiu, "Tough and fully recoverable hydrogels," Journal of Materials Chemistry B, vol. 3, no. 26, pp. 5284-5290, 2015.

[12] L. Zhao, M. D. Weir, and H. H. K. Xu, “An injectable calcium phosphate-alginate hydrogel-umbilical cord mesenchymal stem cell paste for bone tissue engineering," Biomaterials, vol. 31, no. 25, pp. 6502-6510, 2010.

[13] J. Wei, J. Wang, S. Su et al., "3D printing of an extremely tough hydrogel,” RSC Advances, vol. 5, no. 99, pp. 81324-81329, 2015.

[14] H. Park, S. W. Kang, B. S. Kim, D. J. Mooney, and K. Y. Lee, "Shear-reversibly crosslinked alginate hydrogels for tissue engineering," Macromolecular Bioscience, vol. 9, no. 9, pp. 895-901, 2009.

[15] J.-Y. Sun, X. Zhao, W. R. K. Illeperuma et al., "Highly stretchable and tough hydrogels," Nature, vol. 489, no. 7414, pp. 133-136, 2012.

[16] M. C. Darnell, J.-Y. Sun, M. Mehta et al., "Performance and biocompatibility of extremely tough alginate/polyacrylamide hydrogels," Biomaterials, vol. 34, no. 33, pp. 8042-8048, 2013.

[17] J. Li, W. R. K. Illeperuma, Z. Suo, and J. J. Vlassak, "Hybrid hydrogels with extremely high stiffness and toughness," ACS Macro Letters, vol. 3, no. 6, pp. 520-523, 2014.

[18] C. H. Yang, M. X. Wang, H. Haider et al., "Strengthening alginate/polyacrylamide hydrogels using various multivalent cations," ACS Applied Materials \& Interfaces, vol. 5, no. 21, pp. 10418-10422, 2013.

[19] S. Hong, D. Sycks, H. F. Chan et al., "3D printing of highly stretchable and tough hydrogels into complex, cellularized structures," Advanced Materials, vol. 27, no. 27, pp. 40354040, 2015.

[20] A. Nakayama, A. Kakugo, J. P. Gong et al., "High mechanical strength double-network hydrogel with bacterial cellulose," Advanced Functional Materials, vol. 14, no. 11, pp. 11241128, 2004.

[21] J. Wei, S. Su, J. Wang, and J. Qiu, "Imitation proteoglycans improve toughness of double network hydrogels," Materials Chemistry and Physics, vol. 166, pp. 66-72, 2015.

[22] S. Stankovich, D. A. Dikin, G. H. B. Dommett et al., "Graphene-based composite materials," Nature, vol. 442, no. 7100, pp. 282-286, 2006.

[23] J. Wang and J. Qiu, "Luminescent graphene quantum dots: as emerging fluorescent materials for biological application," Science of Advanced Materials, vol. 7, no. 10, pp. 1979-1989, 2015.
[24] J. Wang and J. Qiu, "A review of carbon dots in biological applications," Journal of Materials Science, vol. 51, no. 10, pp. 4728-4738, 2016.

[25] J. Wang and J. Qiu, "A review of organic nanomaterials in photothermal cancer therapy," Cancer Research Frontiers, vol. 2, no. 1, pp. 67-84, 2016.

[26] H. Wang, L. Ma, M. Gan et al., "Design and assembly of reduced graphene oxide/polyaniline/urchin-like mesoporous $\mathrm{TiO}_{2}$ spheres ternary composite and its application in supercapacitors," Composites Part B: Engineering, vol. 92, pp. 405-412, 2016.

[27] J. Shen, B. Yan, T. Li, Y. Long, N. Li, and M. Ye, "Study on graphene-oxide-based polyacrylamide composite hydrogels," Composites Part A: Applied Science and Manufacturing, vol. 43, no. 9, pp. 1476-1481, 2012.

[28] J. Wei, S. Jacob, and J. Qiu, "Graphene oxide-integrated high-temperature durable fluoroelastomer for petroleum oil sealing," Composites Science and Technology, vol. 92, pp. 126-133, 2014.

[29] R. Liu, S. Liang, X.-Z. Tang, D. Yan, X. Li, and Z.-Z. Yu, "Tough and highly stretchable graphene oxide/polyacrylamide nanocomposite hydrogels," Journal of Materials Chemistry, vol. 22, no. 28, pp. 14160-14167, 2012.

[30] S. Das, F. Irin, L. Ma, S. K. Bhattacharia, R. C. Hedden, and M. J. Green, "Rheology and morphology of pristine graphene/polyacrylamide gels," ACS Applied Materials \& Interfaces, vol. 5, no. 17, pp. 8633-8640, 2013.

[31] N. Zhang, R. Li, L. Zhang et al., "Actuator materials based on graphene oxide/polyacrylamide composite hydrogels prepared by in situ polymerization," Soft Matter, vol. 7, no. 16, pp. 72317239, 2011.

[32] H.-P. Cong, P. Wang, and S.-H. Yu, "Highly elastic and superstretchable graphene oxide/polyacrylamide hydrogels," Small, vol. 10, no. 3, pp. 448-453, 2014.

[33] Y. He, N. Zhang, Q. Gong et al., “Alginate/graphene oxide fibers with enhanced mechanical strength prepared by wet spinning," Carbohydrate Polymers, vol. 88, no. 3, pp. 11001108, 2012.

[34] J. Fan, Z. Shi, M. Lian, H. Li, and J. Yin, "Mechanically strong graphene oxide/sodium alginate/polyacrylamide nanocomposite hydrogel with improved dye adsorption capacity," Journal of Materials Chemistry A, vol. 1, no. 25, pp. 7433-7443, 2013.

[35] J. Wang and J. Qiu, "Graphene oxide reinforced double network hydrogel," in ASME 2016 International Mechanical Engineering Congress and Exposition, p. 4, Phoenix, AZ, USA, 2016.

[36] U. Gulyuz and O. Okay, "Self-healing poly(acrylic acid) hydrogels with shape memory behavior of high mechanical strength," Macromolecules, vol. 47, no. 19, pp. 6889-6899, 2014.

[37] C. Chang, M. He, J. Zhou, and L. Zhang, "Swelling behaviors of $\mathrm{pH}$ - and salt-responsive cellulose-based hydrogels," Macromolecules, vol. 44, no. 6, pp. 1642-1648, 2011.

[38] J. Wang, J. Wei, S. Su, and J. Qiu, "Novel fluorescence resonance energy transfer optical sensors for vitamin $B_{12}$ detection using thermally reduced carbon dots," New Journal of Chemistry, vol. 39, no. 1, pp. 501-507, 2015.

[39] D. R. Dreyer, S. Park, C. W. Bielawski, and R. S. Ruoff, “The chemistry of graphene oxide," Chemical Society Reviews, vol. 39, no. 1, pp. 228-240, 2010. 
[40] J. Wei, X. Zhang, J. Qiu, and B. L. Weeks, “Thermal kinetics and thermo-mechanical properties of graphene integrated fluoroelastomer," Journal of Polymer Science Part B: Polymer Physics, vol. 53, no. 23, pp. 1691-1700, 2015.

[41] M. J. McAllister, J.-L. Li, D. H. Adamson et al., "Single sheet functionalized graphene by oxidation and thermal expansion of graphite," Chemistry of Materials, vol. 19, no. 18, pp. 4396-4404, 2007.

[42] P. Cui, J. Lee, E. Hwang, and H. Lee, "One-pot reduction of graphene oxide at subzero temperatures," Chemical Communications, vol. 47, no. 45, pp. 12370-12372, 2011.

[43] D. A. Dikin, S. Stankovich, E. J. Zimney et al., "Preparation and characterization of graphene oxide paper," Nature, vol. 448, no. 7152, pp. 457-460, 2007.

[44] H. Kamata, Y. Akagi, Y. Kayasuga-Kariya, U. Chung, and T. Sakai, "“Nonswellable” hydrogel without mechanical hysteresis," Science, vol. 343, no. 6173, pp. 873-875, 2014.

[45] G. Du, G. Gao, R. Hou et al., "Tough and fatigue resistant biomimetic hydrogels of interlaced self-assembled conjugated polymer belts with a polyelectrolyte network," Chemistry of Materials, vol. 26, no. 11, pp. 3522-3529, 2014.

[46] C. Walter, U. Leichtle, A. Lorenz et al., "Dissipated energy as a method to characterize the cartilage damage in large animal joints: an in vitro testing model," Medical Engineering \& Physics, vol. 35, no. 9, pp. 1251-1255, 2013.

[47] F. Ganji, S. Vasheghani-Farahani, and E. Vasheghani-Farahani, "Theoretical description of hydrogel swelling: a review," Iranian Polymer Journal, vol. 19, pp. 375-398, 2010.

[48] O. Pekcan and S. Kara, "Two-stage swelling of acrylamide gels: a photon transmission study," Journal of Applied Polymer Science, vol. 82, no. 4, pp. 894-906, 2001. 


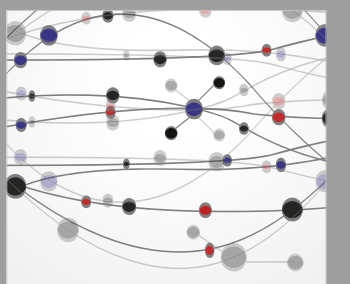

The Scientific World Journal
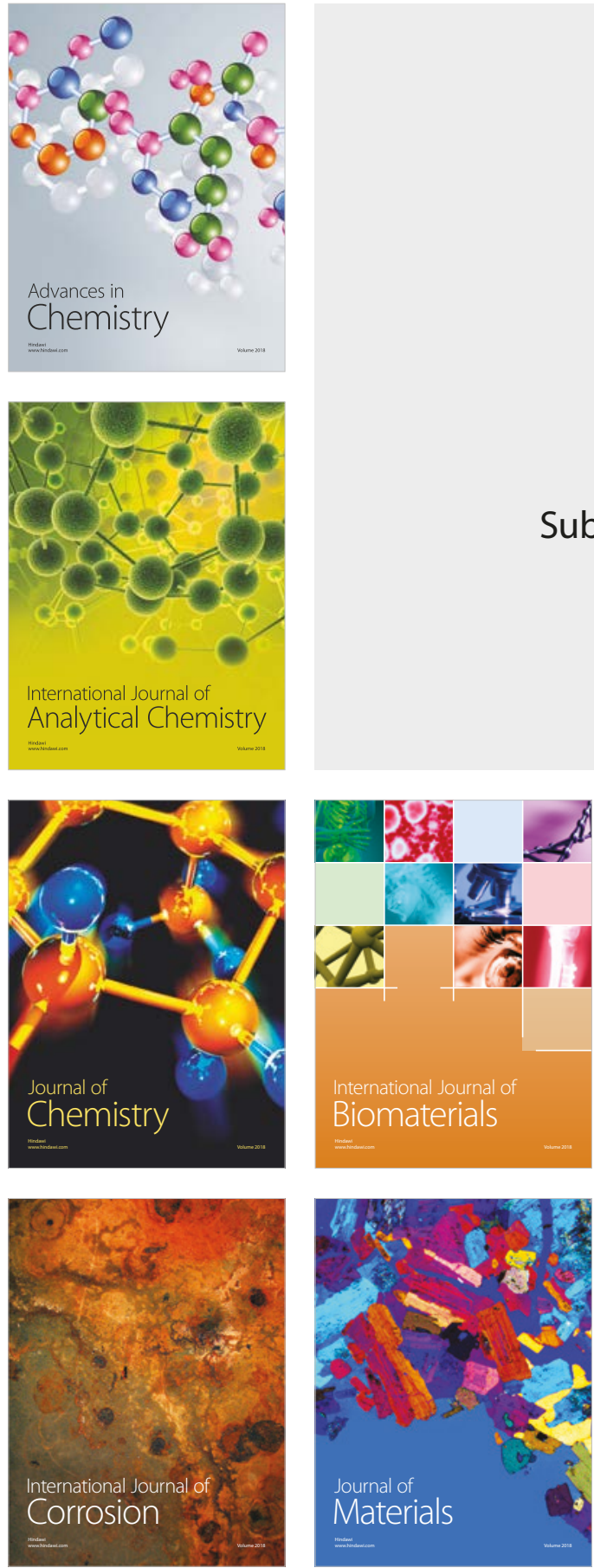

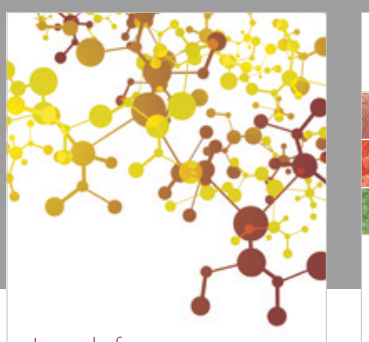

Journal of

Applied Chemistry
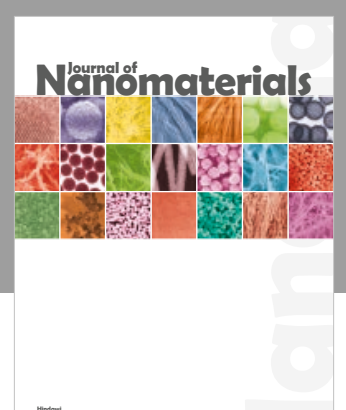

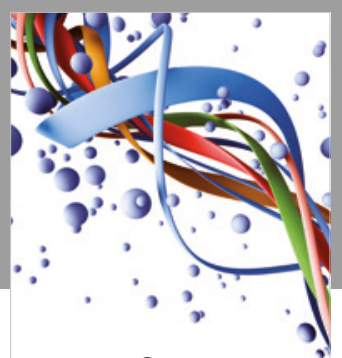

Scientifica

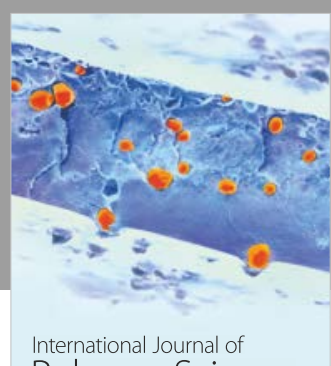

Polymer Science

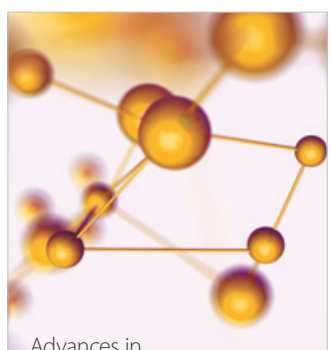

Physical Chemistry
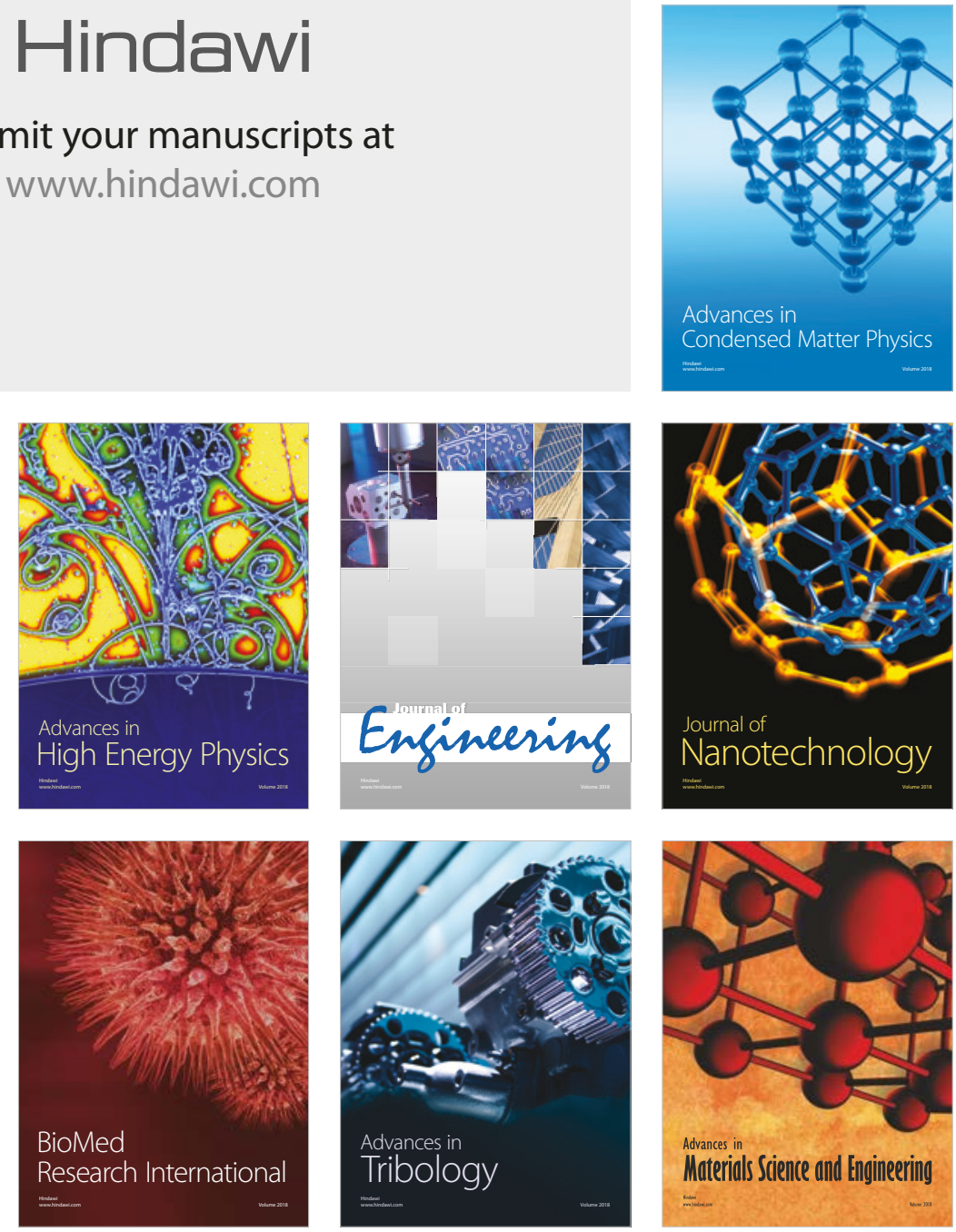